\title{
Quantitative and qualitative differences in the top-down guiding attributes of visual search
}

DOI:

10.1037/xhp0000764

\section{Document Version}

Accepted author manuscript

Link to publication record in Manchester Research Explorer

\section{Citation for published version (APA):}

Hulleman, J. (2020). Quantitative and qualitative differences in the top-down guiding attributes of visual search. Journal of Experimental Psychology: Human Perception and Performance. https://doi.org/10.1037/xhp0000764

\section{Published in:}

Journal of Experimental Psychology: Human Perception and Performance

\section{Citing this paper}

Please note that where the full-text provided on Manchester Research Explorer is the Author Accepted Manuscript or Proof version this may differ from the final Published version. If citing, it is advised that you check and use the publisher's definitive version.

\section{General rights}

Copyright and moral rights for the publications made accessible in the Research Explorer are retained by the authors and/or other copyright owners and it is a condition of accessing publications that users recognise and abide by the legal requirements associated with these rights.

\section{Takedown policy}

If you believe that this document breaches copyright please refer to the University of Manchester's Takedown Procedures [http://man.ac.uk/04Y6Bo] or contact uml.scholarlycommunications@manchester.ac.uk providing relevant details, so we can investigate your claim.

\section{OPEN ACCESS}


Quantitative and qualitative differences in the top-down guiding attributes of visual search

Johan Hulleman

Running Head: Differences in top-down guiding attributes

Word count: 12037

Johan Hulleman

Division of Neuroscience and Experimental Psychology

School of Biological Sciences

The University of Manchester

Manchester M13 9PL

United Kingdom

(C) 2020, American Psychological Association. This paper is not the copy of record and may not exactly replicate the final, authoritative version of the article. Please do not copy or cite without authors' permission. The final article will be available, upon publication, via its DOI: $10.1037 / x h p 0000764$ 
Differences in top-down guiding attributes 2

Keywords: Visual search; Guided Search; guiding attributes; features; subset search; conjunction search; configuration search; Visual Working Memory. 


\begin{abstract}
It is generally assumed that there is a category of undoubted guiding attributes in visual search: colour, motion, orientation. Any differences between these attributes are a matter of degree, rather than kind. This assumption has led to a preferential use of colour in experiments that involve top-down guidance, since it provides the strongest effects. Yet, results observed for colour are considered representative for the other undoubted attributes.

This paper reports seven experiments that compare the top-down guiding strengths of colour, motion and orientation by adding them to a T vs L search. Giving some of the L's a different colour, motion status or orientation should make the T easier to find, since those L's cannot possibly be the target.
\end{abstract}

The results show that whereas adding colour or motion does indeed improve search performance, adding differently oriented L's actually makes search harder, especially on absent trials and even when there are only very few items that could be the target. There were also some subtle differences between colour and motion.

So, rather than a single category of undoubted guiding attributes, there seems to be a clear ranking, with colour at the top and orientation at the bottom. It may therefore be unwise to think that results found with colour will translate one-on-one to other attributes like motion and orientation. 
Differences in top-down guiding attributes 4

Public Significance Statement

Visual search is an important task in both lab and real-world. There are some visual search tasks where the time taken to find the target does not depend on the number of items in the search display. This has led to the proposal that there is a class of attributes (colour, orientation, motion) that guide attention during visual search. Although colour might give the strongest effects, it is nonetheless assumed that what is found for colour also applies to orientation and motion. The current paper shows that this assumption does not hold and that colour really seems to be in a class of its own. 
In visual search, as typically studied in cognitive psychology, the time to find a target or establish its absence usually increases with the number of items in the display. However, there are some search tasks where reaction times are (nearly) independent of the number items $^{1}$. Although opinions differ about the underlying causes (c.f. Treisman \& Gelade, 1980; Wolfe, 1994; Duncan \& Humphreys, 1989; Palmer, Verghese \& Pavel, 2000; Hulleman \& Olivers, 2017a,b), it is generally agreed that there are only a limited number of cases where this independence is observed. In particular, Wolfe and Horowitz $(2004,2017)$ have claimed that only colour, orientation, motion and size are undoubted in their ability to yield flat search slopes and guide attention in general. It is acknowledged that there might be differences in the strength of these attributes, but these are differences in degree, not in kind. For instance, Wolfe (2018, p.581) notes: “[...] all attributes are not equal. It is not clear why one attribute should be more effective than another, but it is clear that color and motion guide attention more effectively than, say, orientation, and that orientation is more effective than various depth cues." This view has been informed by various experimental results that hinted at differences between guidance by orientation and guidance by colour. Wolfe, Friedman-Hill and Bilsky (1994) found that search became more efficient when it was for a part-whole conjunction of the colour of an object with the colour of its part (e.g. a red house with yellow windows amongst red houses with blue windows and blue houses with yellow windows), but Bilsky and Wolfe (1995) failed to find a similar effect when they asked participants to search a part-whole conjunction of orientations (e.g. vertical rectangle with tilted line amongst vertical rectangles with horizontal line and horizontal rectangles with tilted line). Others have also found differences in guidance between colour and orientation. For instance, Poisson and Wilkinson (1992) found that when faced with a choice, participants rather relied on colour than on orientation (see also Sobel \& Cave, 2002; Treisman, 2006). This seems to chime with the results of Olds, Graham and Jones (2009) who found that a preview of the colours in an 
Differences in top-down guiding attributes 6

upcoming conjunction display of colour and orientation improved reaction times relative to a condition without preview, but that a similar preview of the orientations actually led to slower reaction times. More recently, Anderson, Heinke and Humphreys (2010) also reported that search performance in a colour-orientation conjunction task profited more from a colour cue than from an orientation cue. Yet here, even the orientation cue improved performance relative to a neutral cue.

It is important to point out that the specifications of Guided Search do not have any allowance for a difference in guiding strength between orientation and colour. Although Wolfe (1994, p.208) suggests that "In a future version, one might set color activation higher than orientation, on the basis of evidence that color information guides attention more effectively than orientation", this is not actually implemented in Guided Search 2.0. Similarly, the formula for the activation map in Guided Search 4.0 (Wolfe, 2007, p.105) also treats orientation and colour as equivalent, since both get a weight of 1 . Moreover, in relation to a figure containing seven vertical, seven horizontal, thirteen right tilted and six left tilted lines the following point is made (Wolfe, 2007, p.105): "If you were asked to find the targets in Figure 8.5, it would be reasonable to ask, "What targets?" However, if told to find the horizontal items, you can rapidly locate them. Thus, in Figure 8.5, bottom-up salience does not define targets, but efficient search is still possible, guided by top-down information". This statement clearly indicates that orientation is afforded considerable top-down guiding powers in Guided Search 4.0. Furthermore, the suggestion that "Only orientation and color are implemented, but other attributes are presumed to be similar" (Wolfe 2007, p.104) plainly indicates that the undoubted guiding attributes are assumed to differ only in degree, rather than kind. 
This state of affairs has led to an emphasis on the use of colour in experiments, since it creates the largest and most robust effects. However, any effect observed with colour is considered representative for orientation, motion and size. One of the important strands in the literature where the influence of the representativeness assumption of colour can be seen at work is the discussion about Visual Working Memory (VWM) and how its contents can influence top-down guidance of attention. Several of the seminal papers manipulate mainly or even exclusively colour and conjunctions containing colour (e.g. Beck, Hollingworth \& Luck, 2012; Kristjansson, Wang \& Nakayama, 2002; Luck \& Vogel, 1997; Maljkovic \& Nakayama, 1994; Soto, Heinke, Humphreys \& Blanco, 2005; Soto, Heinke, Humphreys, 2006; Van Moorselaar, Theeuwes \& Olivers, 2014; Zhang \& Luck, 2008). Nevertheless, the titles of these reports clearly suggest their results will generalize to other attributes, since they present them in very generic terms. They mention representations, features and conjunctions without any indication that the main manipulation was colour. The current paper will test this assumption that effects of colour are representative for the other undoubted guiding attributes.

The question whether all guiding attributes are created equal is important, since the assumption of equality is at the heart of theories of visual search like FIT and Guided Search $^{2}$. This becomes clear from the illustrations of the theories that have been published over the years. For instance, in what is probably the most up-to-date version of FIT, Figure 1 of Treisman (2006) places orientation on the same footing as colour. Similarly, in what remains the clearest illustration of the input channels used in Guided Search, Figure 2 of Wolfe (1994) clearly implies the equivalence of colour, orientation and other guiding attributes. Moreover, Guided Search assumes that all guiding attributes can be used equally well in top-down fashion (where the observer knows the target they are looking for) and bottom-up fashion (where the observer has to find the odd-one-out). This is another crucial 
assumption that deserves to be tested, since evidence for the status of guiding attribute comes mostly from visual search experiments where the target has a unique attribute value: none of the other search items share this particular attribute value and search slopes tend to be flat.

Yet, guidance is also supposed to be active in searches where the slopes are not flat; for instance in a conjunction search for red vertical amongst red horizontal and green vertical. In Guided Search (Wolfe, 1994; 2007), it is assumed that due to top-down guidance by both colour and orientation this kind of conjunction search yields slopes that are flatter than those in configuration searches like T vs L. In the latter no guidance is assumed possible, since both T and L's are composed of exactly the same two lines and the only difference is their configuration. Consequently, the orientation maps contain no information that can be used to distinguish between target and distractors.

\section{Target}

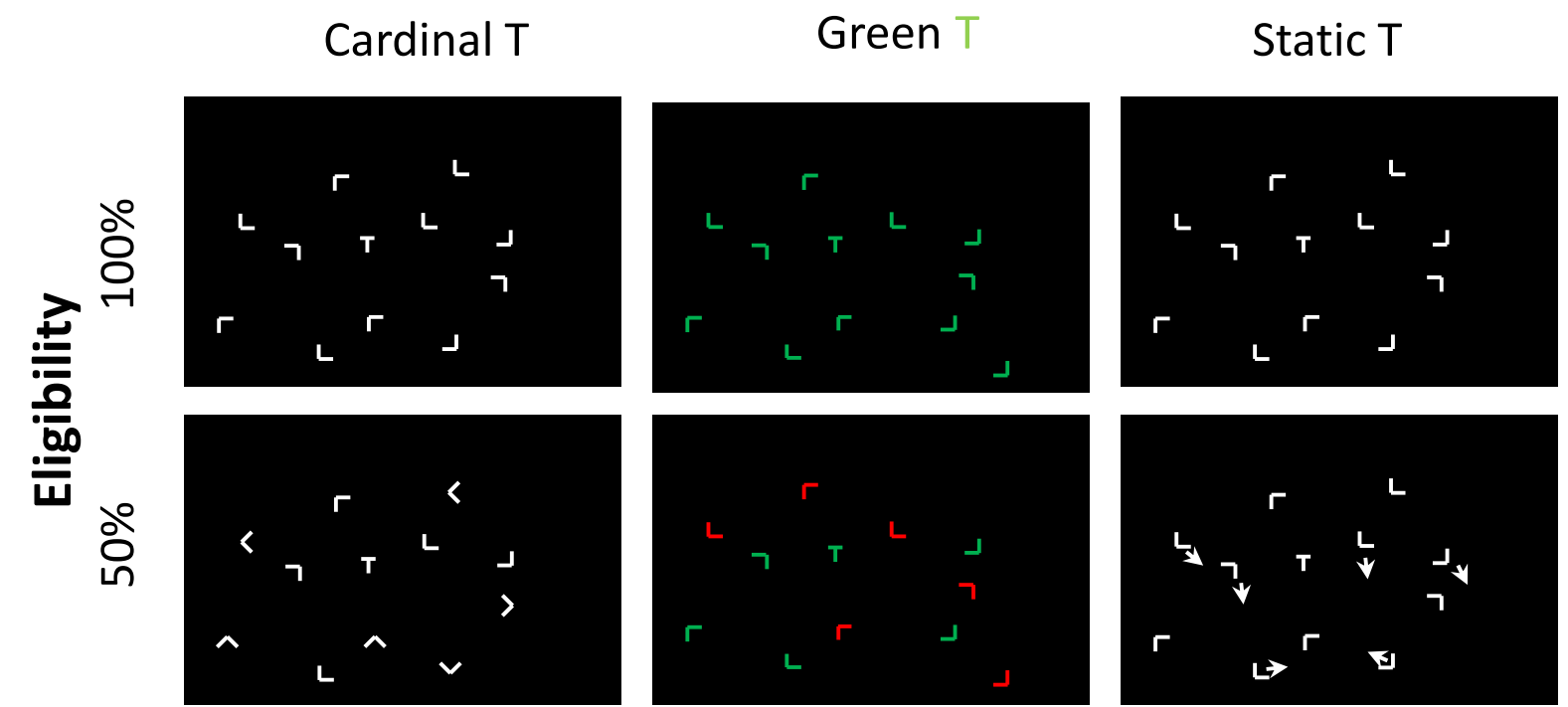

Figure 1. The basic eligibility manipulation used. Top row: 100\% eligibility displays. Here, the target $T$ and distractor Ls all have the same orientation, colour and motion status. So any knowledge about the T will not be helpful. Bottom row: 50\% eligibility displays. Here, half of the items have an orientation, colour or motion status that differs from the target $T$. They can therefore never be the target. This should allow guidance to emerge and result in a smaller effective set size, leading to improved search performance. 
The very fact that $\mathrm{T}$ vs $\mathrm{L}$ search is considered completely unguided means that it is ideally suited for testing the relative strength of the various guiding attributes. Addition of a guiding attribute should improve performance, since an unguided search becomes a guided one. The bigger this improvement is, the stronger the guiding properties of the attribute. The underlying logic is as follows: when some of the search items have an orientation/colour/motion status that is different from the target (e.g. the target is a green T, but some of the distractor L's are red; see Figure 1 for an illustration of the basic manipulation), they become ineligible. Attention should not be guided towards them, since they cannot be possibly be the target. Consequently, the effective set size is reduced and this should result in flatter search slopes.

Recently, Hulleman, Lund and Skarratt (2020) used this approach to test guidance by orientation in a $\mathrm{T}$ vs $\mathrm{L}$ task and reported results that cast doubt on its effectiveness as a guiding attribute in visual search. Essentially, two conditions were compared: $100 \%$ eligibility, where all search items were constructed with the same pair of lines as the target (e.g. a horizontal and a vertical line) and 50\% eligibility where half of the search items was composed of different lines (target and half of the distractors: horizontal and vertical; other half of the distractors: lines rotated 45 degrees clockwise and counter-clockwise). Rather than finding an improvement in search performance when half of the items had an ineligible orientation, Hulleman et al. (2020) actually found a performance decrement. Especially in target-absent trials, reaction times became slower and search slopes steeper. But even in target-present trials there was no improvement in performance. Hulleman et al. (2020) excluded several alternative explanations by showing that performance in response to the same manipulation actually did improve when the search task was made really difficult, and that the performance decrement did not depend on the aspect ratio of the search items. In their discussion, Hulleman et al. (2020) focused on the implications of their result for the 
assumption that visual search always uses a target template that is based on the target in isolation. But the lack of guidance by orientation also raises questions about the equivalence of colour, orientation, motion and size as guiding attributes in visual search, because it contradicts the classic result from Egeth, Virzi and Garbart, (1984). Egeth et al. (1984) found that participants could limit themselves to the small subset of red items (one O and two N's) amongst black O's when they had to search for the red O. Participants' performance improved in both target-present and target-absent trials relative to a condition where the number of red N's and black O's was balanced. (Please note that the title of the paper shows the representativeness assumption for colour in action: it talks about "conjunctively defined targets", rather than about targets defined by a conjunction involving colour). A potentially important difference is that in the task of Hulleman et al. (2020) orientation was incidental to the search task: the target was defined by the fact that it was the only $\mathrm{T}$ in the display rather than by its orientation per se. This was not the case for the shape-colour conjunction used by Egeth et al. (1984). Here, colour was an integral part of the target definition, since there were several O's in the display. So there may be some kind of idiosyncrasy about adding redundant target information that leads to performance decrements.

Given the discrepancies between Egeth et al. (1984) and Hulleman et al. (2020) and the widespread assumption that results for colour will generalise, this paper will explore the communalities and differences between the guiding capacities of motion, colour and orientation. In total, we report seven T vs L searches with colour, orientation and/or motion added to the search display. The first two experiments asked a simple question: When the participants are instructed about the motion status of the $\mathrm{T}$ (Experiment 1) or its colour (Experiment 2), will they become faster at finding it when some of the items in the display have a different motion/colour status ${ }^{3}$ ? 
A performance improvement for motion and/or colour, would strongly imply that there is a qualitative difference between orientation on the one hand and motion/colour on the other. Since this is indeed what was found in Experiments 1 and 2, the next three experiments delved deeper into the influence of eligibility on search times for T's defined by colour (Experiment 3), orientation (Experiment 4) and motion (Experiment 5). Does the beneficial effect of colour and motion kick in immediately? Is there a point where there are so few eligible items that even orientation will improve performance?

Experiments 3-5 found that the performance improvement due to the introduction of ineligible items for colour does indeed start almost immediately when ineligibly coloured items are added. The same holds for motion. For orientation however, even reducing the proportion of eligible items to $17 \%$ still yielded performance deficits in absent trials relative to displays where all items were eligible.

The final two experiments explored the interaction between orientation on the one hand and motion and colour on the other. Is the performance detriment due to the introduction of ineligible orientations unavoidable, or can colour (Experiment 6) and/or motion (Experiment 7) overcome its negative effects?

The results of Experiments 6 and 7 indicate that colour is more impervious to the negative influence of orientation than motion. This then leads to the conclusion that rather than a single category of unguided attributes that contains colour, orientation and motion there is a clear ranking in guidance strength with colour at the top and orientation at the bottom.

\section{General Method}

This paper measures the relative strength of top-down guidance by colour, orientation and motion by adding them to T vs L search. According to Guided Search (Wolfe, 1994; 2007) 
this type of search is completely unguided. Both $\mathrm{T}$ and $\mathrm{L}$ are constructed from the same kind of lines and effortful item-by-item inspection is needed to find the target. The addition of colour, orientation, or motion should therefore improve performance. By telling the participants the colour/orientation/motion of the target, they should be able to guide their attention in a top-down fashion towards the eligible items that have this colour/orientation/motion and away from the ineligible items that have a different colour/orientation/motion. Effectively, this reduces the set size and should therefore result in faster reaction times and fewer errors. (See Figure 1 for an illustration of the basic manipulation). We instructed the participants to search for the red $\mathrm{T}$, the moving $\mathrm{T}$, or the tilted $\mathrm{T}$ and assessed whether they could restrict themselves to this subset and ignore the green L's, the static L's and the upright L's.

\section{Participants}

Undergraduates (UG) and postgraduates (PG) from the Universities of Hull and Manchester were recruited for these experiments, with sixteen participating in each of the seven experiments. Please see Table 1 for a detailed breakdown of the demographics. All gave their written informed consent, participated in return for course credit and were naïve to the purpose of the experiment. None participated in more than one experiment. 
Table 1. Demographics of participants

\begin{tabular}{|c|c|c|c|c|c|c|}
\hline Experiment & $\begin{array}{l}\mathrm{N} \\
(\mathrm{UG} / \mathrm{PG})\end{array}$ & University & $\begin{array}{l}\text { Mean age } \\
\text { (years) }\end{array}$ & $\begin{array}{l}\text { Age range } \\
\text { (years) }\end{array}$ & Female/Male & $\begin{array}{l}\text { Left-handers } \\
\text { (female/male) }\end{array}$ \\
\hline $1-$ Motion & $16 / 0$ & Hull & 20 & $18-26$ & $9 / 7$ & $1 / 1$ \\
\hline $2-$ Colour & $16 / 0$ & Manchester & 20 & $18-24$ & $14 / 2$ & $2 / 0$ \\
\hline 3 -Colour & $16 / 0$ & Manchester & 20 & $18-26$ & $15 / 1$ & $3 / 1$ \\
\hline 4 - Orientation & $16 / 0$ & Hull & 21 & $18-33$ & $13 / 3$ & $0 / 0$ \\
\hline 5 - Motion & $16 / 0$ & Hull & 20 & $18-27$ & $12 / 4$ & $0 / 0$ \\
\hline $6-$ Colour + & $16 / 0$ & Manchester & 19 & $18-20$ & $15 / 1$ & $0 / 0$ \\
\hline \multicolumn{7}{|l|}{ Orientation } \\
\hline $7-$ Motion + & $13 / 3$ & Manchester & 21 & $18-25$ & $12 / 4$ & $1 / 1$ \\
\hline Orientation & & & & & & \\
\hline
\end{tabular}

\section{Power analysis}

The effect of interest in these experiments is that of eligibility. The results in Hulleman et al. (2020) show that the effect size of eligibility is in the order of $\eta_{\mathrm{p}}{ }^{2}$. 60 . Using this value as an estimate in G*Power 3.1.3 (Faul, Erdfelder, Buchner \& Lang, 2009), all experiments reported had a power of $>.99$ with the 16 participants used. Hulleman et al. (2020) also found that the effect of eligibility tends to be larger in target absent trials than in target present trials. In their Experiment 4, the effect size for the interaction between eligibility and target was in the order of $\eta_{\mathrm{p}}{ }^{2} \sim .55$. This means that all our experiments had a power of $>.99$ for this interaction as well. 


\section{Stimuli}

The same custom-written software presented and recorded responses across all experiments. The three experiments in Hull (Experiments 1, 4 and 5) used an Iiyama 454 Vision Master Pro CRT controlled by a GeForce 6800 graphics card. Experiment 2 (Manchester) was run on a BenQ XL2420B LCD display controlled by a Dell Optiplex GX-620 PC with Intel 82945G Express integrated graphics. The other three Manchester experiments (3, 6 and 7$)$ used a ViewSonic VX2268WM LCD display controlled by a Quadro FX580 graphics card. The resolution was $800 \times 600$ pixels for all experiments. All monitors used a 4:3 aspect ratio, with a refresh rate of $75 \mathrm{~Hz}$.

The search items consisted of two lines $\left(0.1^{\circ} \mathrm{x} 0.96^{\circ}\right)$ on a black background. All lines were white, unless the target was defined by colour. In all experiments, the target was a $\mathrm{T}\left(0.96^{\circ} \mathrm{x}\right.$ $\left.0.96^{\circ}\right)$ and the distractors were L's $\left(0.96^{\circ} \times 0.96^{\circ}\right)$. Targets defined by colour could by either red or green ${ }^{4}$; targets defined by motion were either static $(0.0 \%)$ or moving $(7.2 \%)$; and targets defined by the orientation of their constituting lines where either constructed from a cardinal line set (horizontal line + vertical line) or from a diagonal line set (left diagonal line + right diagonal line). In the colour and motion experiments, all items were drawn with lines from the cardinal line set. This meant that the distractor L's had four possible orientations: $90^{\circ}$, upright, $+90^{\circ}$, and $+180^{\circ}$. In the orientation experiments, the distractor L's could also be drawn with lines from the diagonal line set resulting in four different possible orientations: $135^{\circ},-45^{\circ}, 45^{\circ}$, and $135^{\circ}$. Items were randomly placed in a virtual rectangle $\left(29.0^{\circ} \times 19.3^{\circ}\right)$, with a minimum distance of $1.45^{\circ}$ between them. In the experiments where items moved, the direction of travel was randomly chosen. Whenever two moving items reached the minimum distance, they would bounce off each other in an elastic collision. Moving items would also bounce off static items, but the static items themselves always remained in the same position. 


\section{Design and Analysis}

The design was similar across all experiments. There were four independent variables: two that constitute the basic factors of visual search, display size and target (present in $50 \%$ of the trials), and two that were the main interest of the experiments: target type (the colour/motion/orientation that defines the target) and eligibility (the proportion of distractors of the same type as the target). The focus of the analyses reported here will be most prominently on eligibility and its interactions, since this factor measures the changes in performance as a function of the proportion of eligible items in the display. Eligibility varied from $100 \%$ (all items in the display had the same colour/motion/orientation as the target) down to $17 \%$ (only two or three of the distractors shared their colour/motion/orientation with the target; all other distractors had the opposite colour/motion/orientation). The factors were fully crossed in all experiments, and were all analysed with Greenhouse-Geisser corrected four-way repeated measures ANOVAs. The factors target (absent/present) and target type (colour: red/green; motion: static/moving; orientation: cardinal/diagonal) always had two levels. For the factors eligibility and display size the number of levels varied across experiments. The exact number of items in the search display depended on the proportions of eligibility used (e.g. for $20 \%$ eligibility the number of items has to be a multiple of five, but for $17 \%$ eligibility it has to be a multiple of six). There were either 20 or 25 trials per cell of the design. The exact levels for eligibility and display size and the exact number of trials are listed for each experiment separately. 


\section{Analysis Strategy}

The basic analysis strategy was to find a main effect of eligibility which indicates that changing eligibility changes the reaction times/error rates. The effect of eligibility on reaction times is usually more pronounced for absent trials than for present trials. This would be signalled by a significant eligibility $\mathrm{x}$ target interaction. The effect of eligibility can also change search slopes (eligibility $\mathrm{x}$ display size) and the type of target used (eligibility $\mathrm{x}$ target type). Higher order interactions are of course also possible. To make the effects of eligibility interpretable when there are higher order interactions in the four-way ANOVA, the analysis was split into a three-way ANOVA for target-absent trials and a three-way ANOVA for target-present trials. This was usually enough to get an outcome that makes it possible to characterize the effect of eligibility. Only when there was a three-way interaction (which indicates that the effect of eligibility depends both on display size and target type) was the analysis split further by target type. So, the detailed analysis happens in the three-way ANOVAs and readers in a hurry may go straight to them. The higher order interactions in the four-way ANOVA are reported to guarantee that any differences between the two three-way ANOVAs (e.g. the effect of eligibility is larger in absent than in present trials) are consistent across participants. Please note that the type of task used ( $T$ vs L) mandates that there are significant effects of target, display size and an interaction between them. These merely indicate that the search slopes are not flat, something that is well-known (e.g. Wolfe, 1994). 


\section{Data Cleaning}

For all experiments, trials with reaction times further than 2.5 SD's from the cell mean were removed. All remaining data was used for the error analysis, but only correct trials were used for the reaction time analysis. The exact percentage of trials removed and any other issues with the data will be reported at each experiment. Please note that the broad conclusion from this paper that there is a clear ranking in guidance strength with colour at the top and orientation at the bottom, does not critically depend on the cleaning procedure used.

Procedure

Participants were informed about the defining attribute of the target and were instructed to find the T as quickly and accurately as possible. Responses were collected either via the " $Z$ " and "M" keys on a UK-keyboard (Experiment 2), or via the left and right trigger button of a Sidewinder Gamepad. Participants received one or several practice sessions (10 trials per session) until they were considered trained enough to start the main experiment. The trials were blocked by target type (colour: red/green, motion: static/moving, orientation cardinal/diagonal), but randomized across the other factors. Target type itself was counterbalanced across participants. Depending on the experiment, there was a self-paced break after either every 25 or 50 trials. Half-way during each experimental session there was a bigger break, where participants received new instruction and practice trials for the second block with the new target type. 
Experiment 1 - Guidance by Motion

\section{Method}

The target was defined by its motion status and was either static or moving with $7.2 \%$. The eligibility levels were $100 \%$ and $50 \%$, the display sizes used were 12 and 18 , and there were 25 trials per cell. (See Figure 2 for an overview of the stimulus conditions)

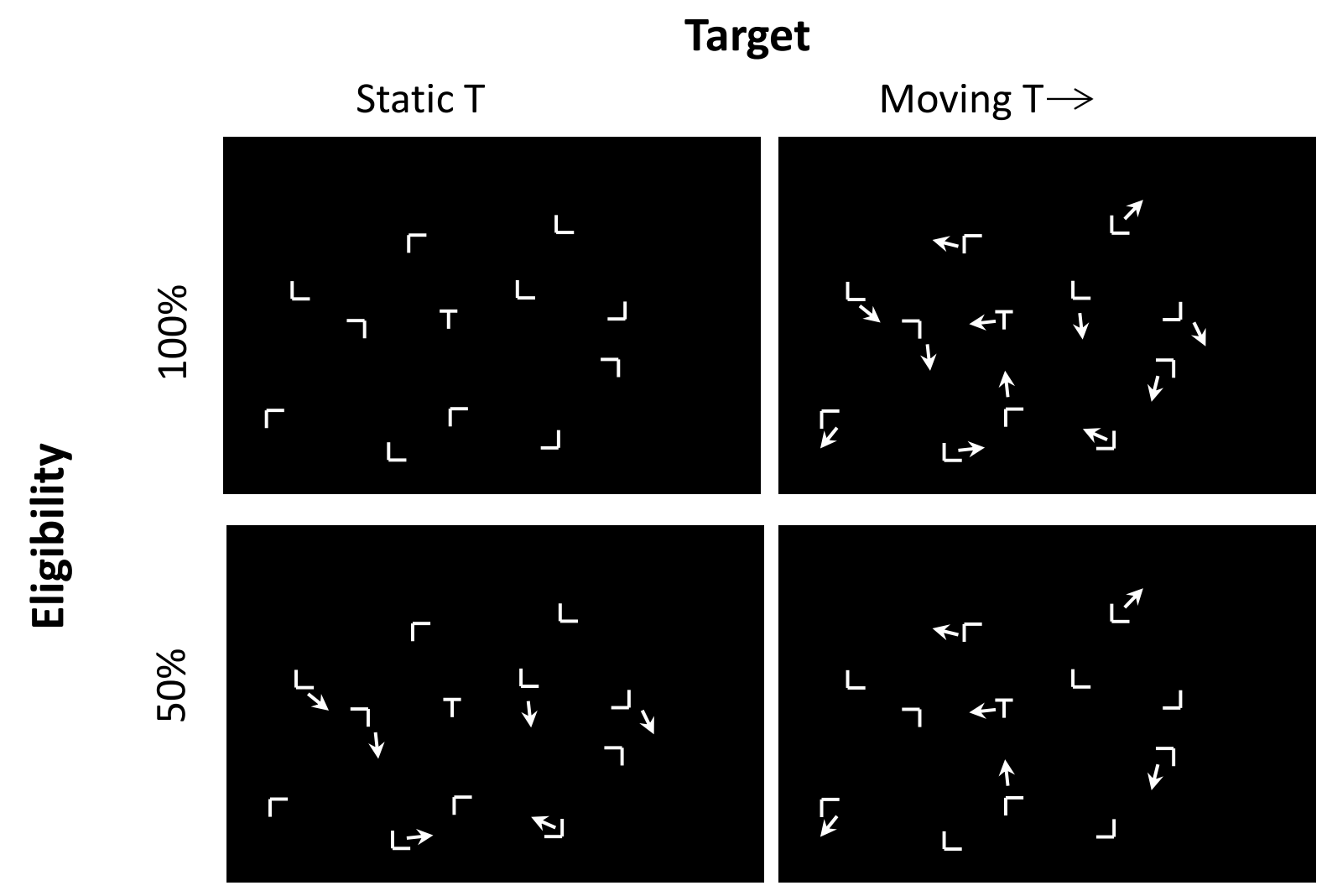

Figure 2. Manipulation used in Experiment 1. Top Row: 100\% eligibility displays, Bottom Row: 50\% eligibility displays. Left: search for a static T. Right: Search for a moving T. 
Results

Summary

Reducing the number of eligible items resulted in faster reaction times. Reaction times improved in both target-absent and target-present trials, but this improvement was much clearer in target-absent trials. The error proportions stayed relatively constant. The reaction times are shown in Figure 3, the error rates are listed in Table 2 and the slopes can be found in Table A1 (appendix) . Outlier removal excluded $1.9 \%$ of the trials.

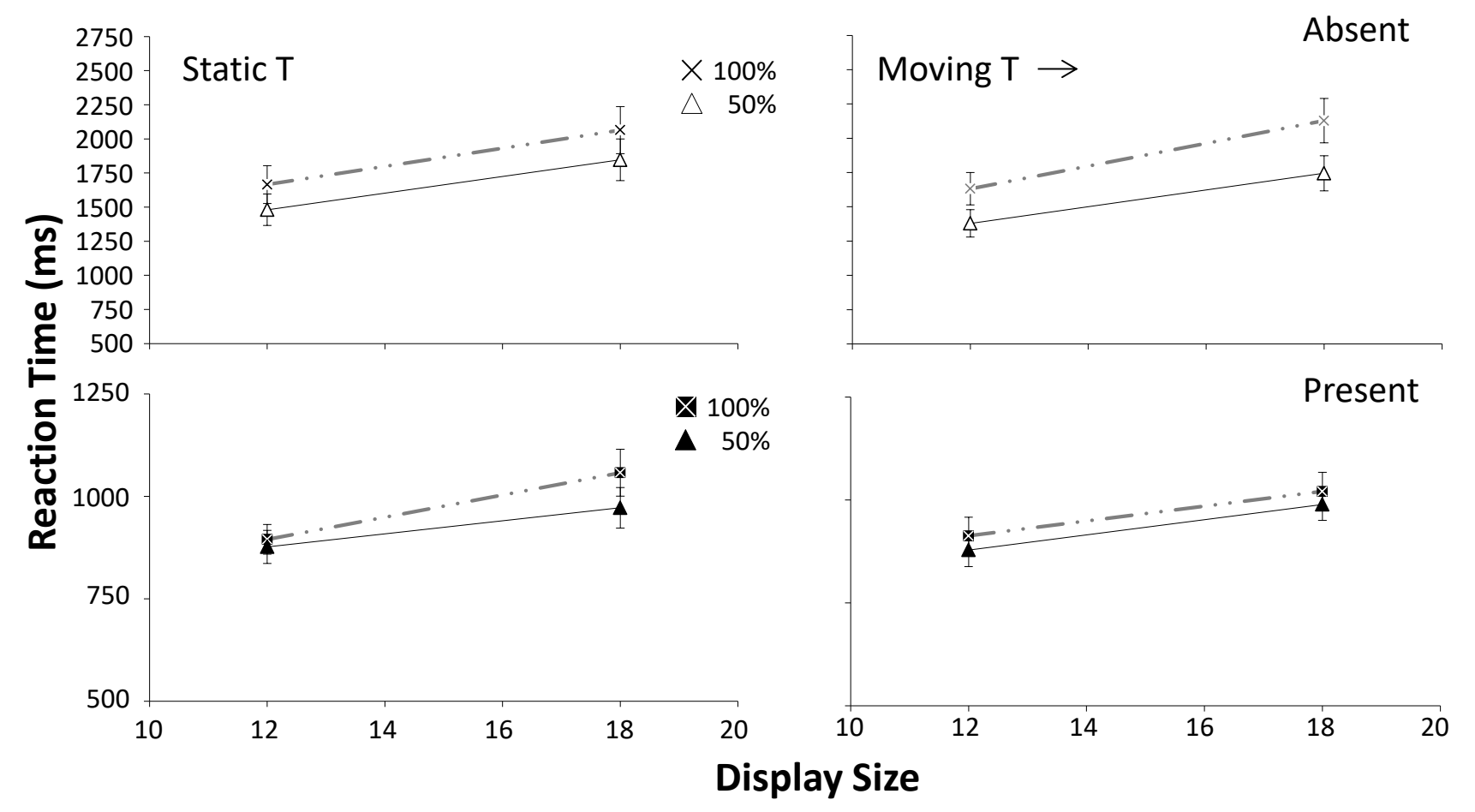

Figure 3. Results of Experiment 1. Reaction times as a function of eligibility, target type, display size and target presence. Left: static T target; Right: Moving T target. Top: absent trials; Bottom: present trials. The grey dashed line is the 100\% eligibility condition. Triangles are the 50\% eligibility condition. The error bars indicate the between-subject standard error of the mean. Slopes can be found in Table Al in the appendix. 


\section{Reaction Times}

\section{Four-way ANOVA}

The repeated measures ANOVA found a four-way interaction between eligibility, motion, display size and target $F(1,15)=5.02, p=.041, \eta_{\mathrm{p}}{ }^{2}=.25$. Because of this interaction, there were separate analyses for target-absent trials and target-present trials.

\section{Three-way ANOVAs}

For the target-absent trials, a three-way repeated measures ANOVA (eligibility $\mathrm{x}$ motion $\mathrm{x}$ display size) yielded a significant main effect of eligibility $F(1,15)=42.438$, $p<.001, \eta_{\mathrm{p}}{ }^{2}=.74$, with faster reaction times for $50 \%$ eligibility than for $100 \%$ eligibility (1613 ms vs. $1873 \mathrm{~ms})$. The interaction between eligibility and motion $F(1,15)=7.40, p=.016$, $\eta_{\mathrm{p}}{ }^{2}=.33$ indicates that the improvement in reaction times due to reduction in eligibility was larger when the target was moving (from $1880 \mathrm{~ms}$ down to $1562 \mathrm{~ms}$ ) than when the target was static (1865 ms down to $1663 \mathrm{~ms})$. There was also a marginal interaction between eligibility and display size $F(1,15)=4.87, p=.043, \eta_{\mathrm{p}}{ }^{2}=.25$, with a larger improvement due to reduced eligibility for 20 item displays (2096 ms down to $1796 \mathrm{~ms}$ ) than for 12 item displays (1649 ms down to $1430 \mathrm{~ms}$ ). There was no three-way interaction $F(1,15)=1.87, p=.19$, $\eta_{\mathrm{p}}^{2}=.11$.

For the target-present trials a similar three-way repeated measures ANOVA merely found a main effect of eligibility $F(1,15)=5.22, p=.037, \eta_{\mathrm{p}}{ }^{2}=.26$, with reaction times for $50 \%$ eligibility faster than reaction times for $100 \%$ (929 ms vs $971 \mathrm{~ms}$ ). None of the interactions involving eligibility was significant (all $F^{\prime} \mathrm{s}(1,15)<1.47$, all $p$ 's $>.24$, all $\eta_{\mathrm{p}}{ }^{2}{ }^{\prime}<.09$ ). 


\section{Errors}

There were no effects whatsoever for target-absent trials. For target present trials there was no effect of eligibility when the target was static. But when the target moved, the error rate increased for when eligibility was decreased to $50 \%$. Please see the appendix for more detail. Table 2 Error Rates Experiment 1

\begin{tabular}{|c|c|c|c|c|c|c|c|c|}
\hline & \multicolumn{8}{|l|}{ Motion } \\
\hline & \multicolumn{4}{|l|}{ Static T } & \multicolumn{4}{|l|}{ Moving T } \\
\hline & \multicolumn{3}{|l|}{ Target } & & \multicolumn{3}{|l|}{ Target } & \\
\hline & \multicolumn{2}{|l|}{ Absent } & \multicolumn{2}{|l|}{ Present } & \multicolumn{2}{|l|}{ Absent } & \multicolumn{2}{|l|}{ Present } \\
\hline & 12 Items & 18 Items & 12 Items & 18 Items & 12 Items & $\overline{18}$ Items & 12 Items & 18 Items \\
\hline Eligibility & & & & & & & & \\
\hline $100 \%$ & .01 & .02 & .02 & .03 & .01 & .01 & .02 & .01 \\
\hline $50 \%$ & .00 & .01 & .02 & .02 & .01 & .01 & .04 & .03 \\
\hline
\end{tabular}

Experiment 2 - Guidance by Colour

\section{Participants}

\section{Method}

The target was defined by its colour status (red/green). There were four levels of eligibility $(100 \%, 75 \%, 50 \%, 25 \%)$ and the display sizes used were 12 and 20. Per cell of the design there were 20 trials. (See Figure 4 for an overview of the stimulus conditions)

Results

\section{Summary}

For target-absent trials there was already a reaction time improvement when eligibility fell to $75 \%$. For target-present trials the reaction time improvement only became manifest for $50 \%$ eligibility displays. Again, the error rates remained fairly constant. The reaction times are shown in Figure 5, the error rates are listed in Table 3 and the slopes can be found in Table A2 (appendix). Outlier removal excluded $1.9 \%$ of the trials 


\section{Target}
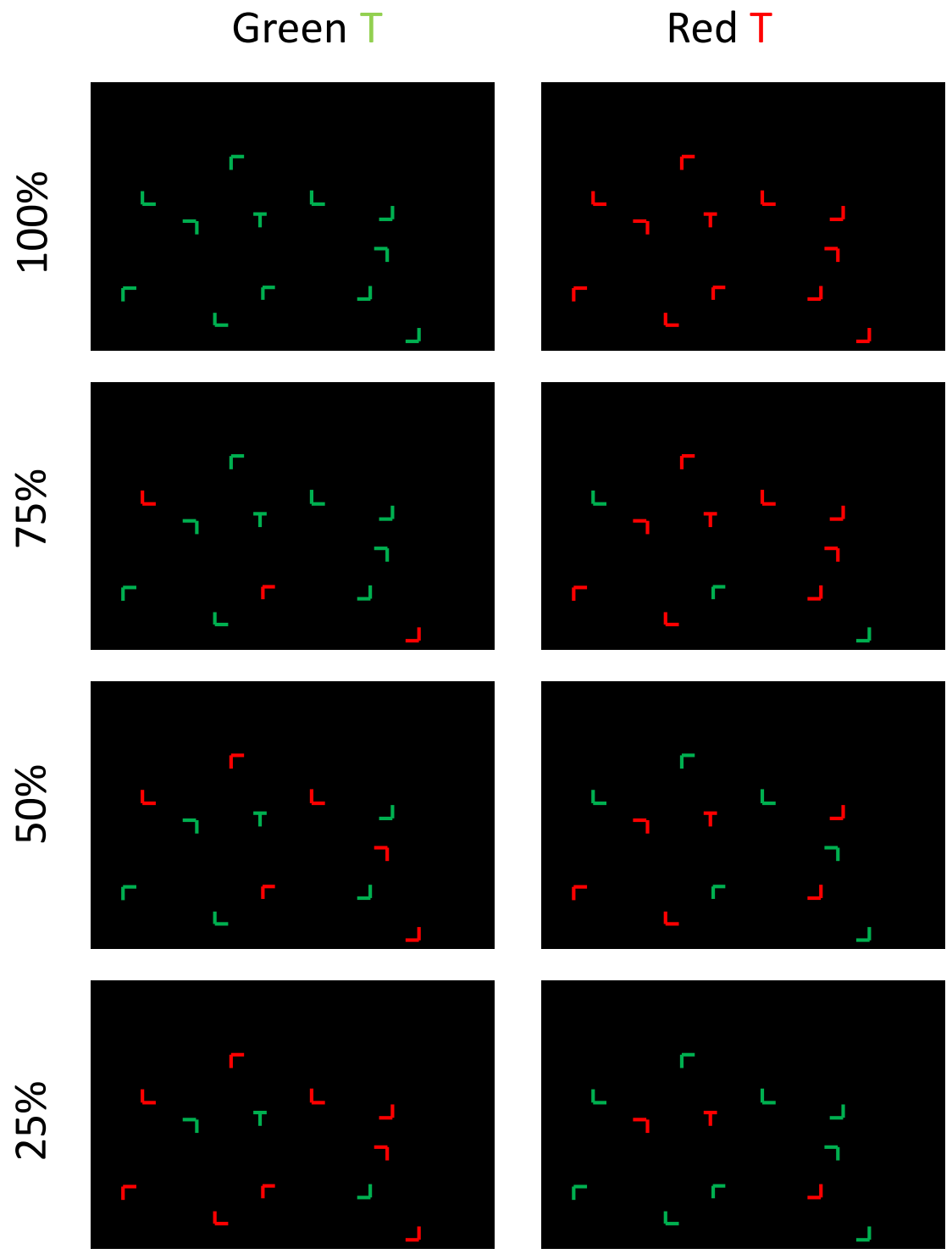

Figure 4 Manipulation used in Experiment 1. From the top row: 100\% eligibility displays; 75\% eligibility displays; $50 \%$ eligibility displays; and 25\% eligibility displays. Left: search for a green T. Right: Search for a red T.

\section{Reaction Times}

\section{Four-way ANOVA}

There was a main effect of eligibility $F(1.30,19.45)=98.48, p<.001, \eta_{\mathrm{p}}{ }^{2}=.87$, two-way interactions between eligibility and display size $F(2.32,34.72)=18.09, p<.001, \eta_{\mathrm{p}}{ }^{2}=.55$ and between eligibility and target $F(1.19,17.88)=61.62, p<.001, \eta_{\mathrm{p}}{ }^{2}=.80$ and a three-way 
interaction between eligibility, display size and target $F(2.14,32.02)=7.08, p=.002, \eta_{\mathrm{p}}{ }^{2}=.32$.

Because of the interactions we split the analysis by target.

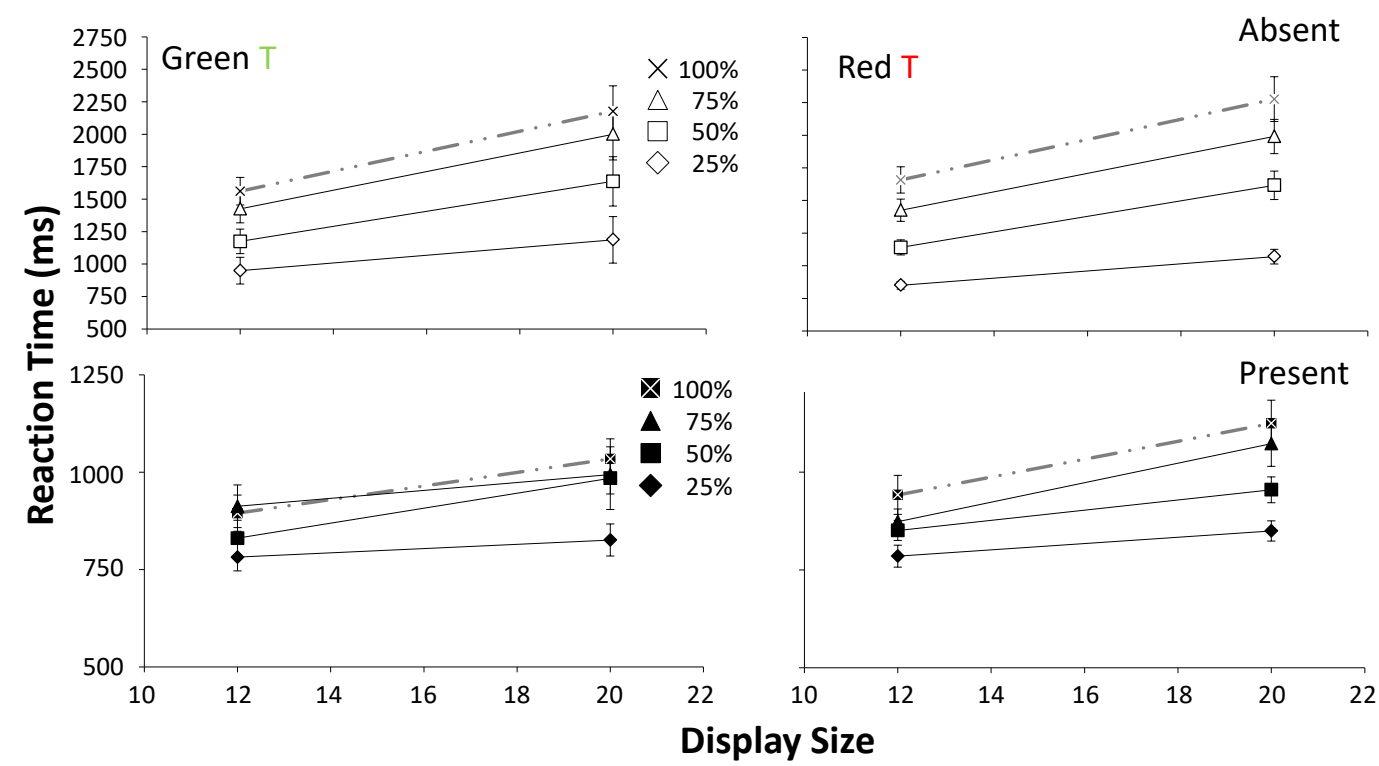

Figure 5. Results of Experiment 2. Reaction times as a function of eligibility, target type, display size and target presence. Left: Green T target; Right: Red T target. Top: absent trials; Bottom: present trials. The grey dashed line is the 100\% eligibility condition. Triangles, squares and diamonds are the $75 \%, 50 \%$, and $25 \%$ eligibility conditions, respectively. The error bars indicate the between-subject standard error of the mean. Slopes can be found in Table A2 in the appendix.

\section{Three-way ANOVAs}

For target-absent trials, a three-way repeated measures ANOVA (eligibility x colour $\mathrm{x}$ display size) found a main effect of eligibility $F(1.16,17.44)=91.62, p<.001, \eta_{\mathrm{p}}{ }^{2}=.86$, with progressively faster reaction times for lower eligibilities (1918 ms -> $1710 \mathrm{~ms}$-> $1392 \mathrm{~ms}$-> 1014 ms for $100 \%, 75 \%, 50 \%$ and $25 \%$, respectively). A planned contrast revealed that reaction times for $75 \%$ eligibility were already faster than $100 \%: F(1,15)=22.40, p<.001$, $\eta_{\mathrm{p}}{ }^{2}=.60$. There was also an interaction between eligibility and display size $F(2.23$, $33.38)=16.49, p<.001, \eta_{\mathrm{p}}{ }^{2}=.52$, indicating that the improvement in reaction times as a function of eligibility was larger at 20 items (2227 ms ->1995 ms ->1627 ms ->1128 ms) than

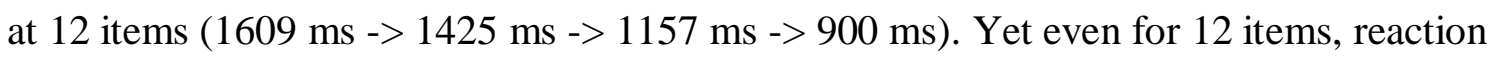
times improved when eligibility was reduced from $100 \%$ to $75 \%: t(15)=4.95, p<.001, \mathrm{~d}=1.24$. 
For target-present trials a similar three-way repeated measures ANOVA also found a main effect of eligibility $F(1.95,29.17)=27.27, p<.001, \eta_{\mathrm{p}}{ }^{2}=.65$. As before, reaction times became faster as eligibility was reduced (999 ms -> $963 \mathrm{~ms}$-> $905 \mathrm{~ms} \mathrm{->} 811 \mathrm{~ms}$ ). However, only when eligibility dropped to $50 \%$ did the improvement in reaction times relative to $100 \%$ become manifest $F(1,15)=10.41, p=.006, \eta_{\mathrm{p}}{ }^{2}=.41$. Again, there was an interaction between eligibility and display size $F(2.33,34.94)=3.30, p=.042, \eta_{\mathrm{p}}{ }^{2}=.18$ with larger reaction time reductions for 20 items $(1079 \mathrm{~ms}$-> $1033 \mathrm{~ms}->970 \mathrm{~ms}$-> $838 \mathrm{~ms})$ than for 12 items $(918 \mathrm{~ms}$

-> $893 \mathrm{~ms}$-> $841 \mathrm{~ms}$-> $784 \mathrm{~ms})$. For both display sizes, reaction times only became significantly faster at 50\% eligibility $[t(15)=2.45, p=.027, \mathrm{~d}=.61$ and $t(15)=2.72, p=.016$, $\mathrm{d}=.68$ for 12 and 20 items, respectively].

\section{Errors}

For target absent trials there were no effects of eligibility. For target present trials the $50 \%$ eligibility condition had fewer errors than $100 \%$ eligibility, but only for 20 item displays. Please see the appendix for more detail.

Table 3 Error Rates Experiment 2

\begin{tabular}{|c|c|c|c|c|c|c|c|c|}
\hline & \multicolumn{8}{|l|}{ Colour } \\
\hline & \multicolumn{4}{|l|}{ Green $\mathrm{T}$} & \multicolumn{4}{|l|}{ Red T } \\
\hline & \multicolumn{3}{|l|}{ Target } & & \multicolumn{4}{|l|}{ Target } \\
\hline & \multicolumn{2}{|l|}{ Absent } & \multicolumn{2}{|l|}{ Present } & \multicolumn{2}{|l|}{ Absent } & \multicolumn{2}{|l|}{ Present } \\
\hline & 12 Items & 20 Items & 12 Items & 20 Items & 12 Items & 20 Items & 12 Items & 20 Items \\
\hline Eligibility & & & & & & & & \\
\hline $100 \%$ & .01 & .01 & .04 & .05 & .01 & .00 & .03 & .05 \\
\hline $75 \%$ & .00 & .01 & .02 & .04 & .00 & .01 & .02 & .05 \\
\hline $50 \%$ & .00 & .00 & .02 & .02 & .01 & .02 & .05 & .03 \\
\hline $25 \%$ & .01 & .00 & .06 & .04 & .00 & .01 & .03 & .04 \\
\hline
\end{tabular}

\section{Discussion of Experiments 1 and 2}

Contrary to the results for orientation in Hulleman et al. (2020), there were improvements in performance when colour or motion status was added to a T vs L display as indicators of eligibility. Whereas Hulleman et al. (2020) found that giving $50 \%$ of the distractors a 
different orientation yielded worse performance in target-absent trials and no improved performance in target-present trials, here we found that making distractors ineligible by giving them a different colour or a different motion status improved performance on both. It is especially notable that the biggest improvements were found for target-absent trials, since this is where Hulleman et al. (2020) found the largest performance decrements when the guiding attribute was orientation. The current results also put to rest the potential objection that Hulleman et al. (2020) may have found no improvement on target-present trials due to a floor effect. Here, it becomes clear that there certainly is scope for improvement in performance from $100 \%$ eligibility when searching for T amongst L. Yet it should also be pointed out that neither colour nor motion status is perfect in determining eligibility. Had they been, then the reaction times for the larger display size at $50 \%$ eligibility would have been faster than for the smaller display size at $100 \%$ eligibility, because there are fewer eligible items in the $50 \%$ display.

The contrasts in the guiding capabilities of colour, motion and orientation observed here and in Hulleman et al. (2020) are quite marked. The next three experiments therefore tested a wider range of eligibilities to investigate the limits of guidance by colour, orientation and motion in more detail. 
Experiment 3 - Guidance by Colour

\section{Method}

The target was defined by its colour status. The eligibilities were $20,40,60,80$, and $100 \%$, the display sizes were 10 and 20 and there were 25 trials per cell of the design. (See Figure 6 for an overview of the stimulus conditions).

\section{Results}

Summary

The reaction time data shows that by the time that eligibility was reduced to $60 \%$, there was a distinct reaction time advantage for both target-absent and target-present trials. There we no systematic changes in error rates. The reaction times are shown in Figure 7, the error rates are listed in Table 4 and the slopes can be found in Table A3 (appendix). Outlier removal excluded $1.8 \%$ of the trials (Additionally, the final 42 trials from the red target block for one participant were lost due to a computer failure)

\section{Reaction times}

\section{Four-way ANOVA}

The four-way repeated measures ANOVA on reaction times with colour, eligibility, display size and target as factors yielded a significant four-way interaction between colour, eligibility, display size and target $F(2.90,43.53)=2.91, p=.047, \eta_{\mathrm{p}}{ }^{2}=.162$. Because of this interaction, the analysis was split along the target dimension. 
Differences in top-down guiding attributes 27

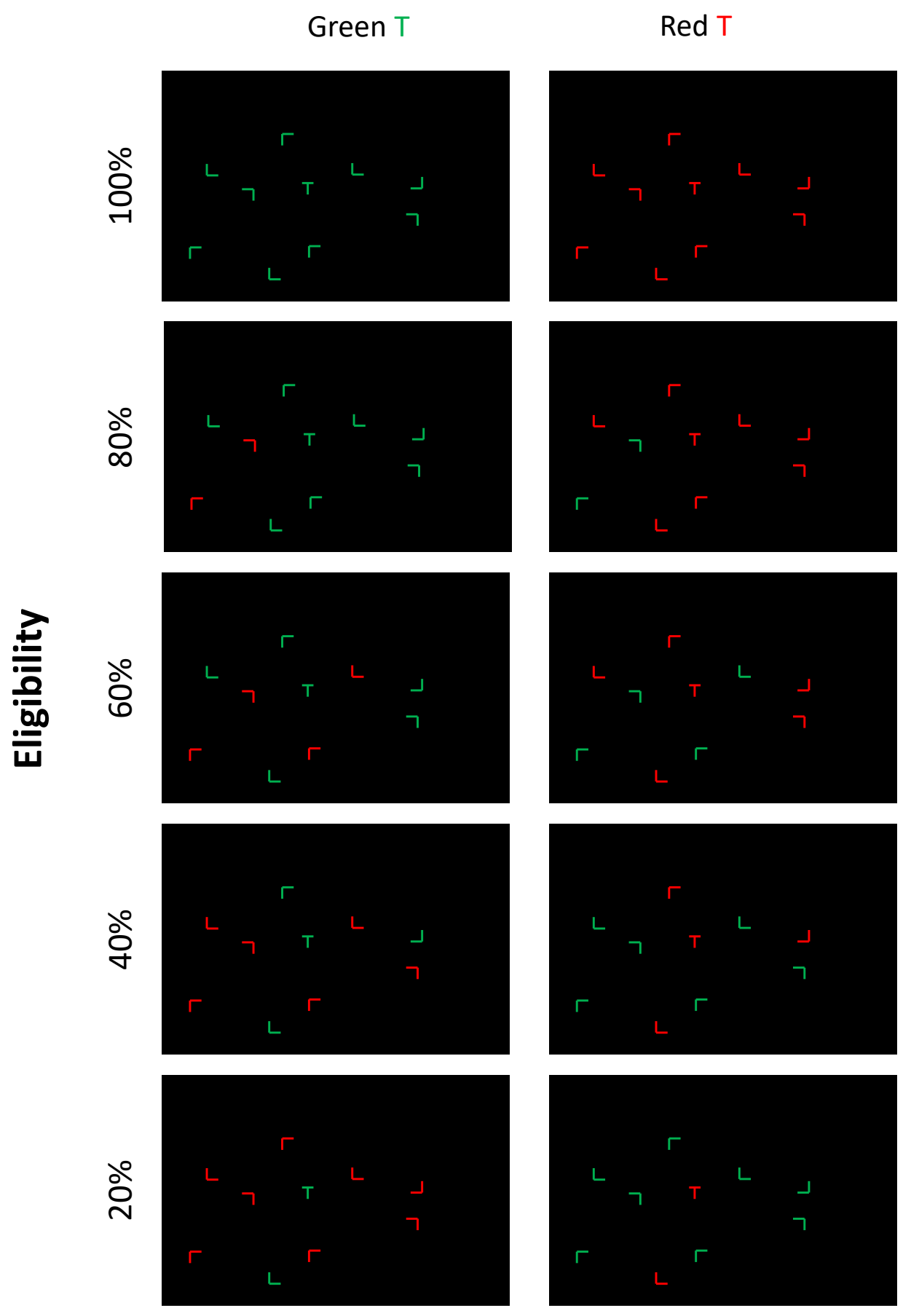

Figure 6. Manipulation used in Experiment 3. From the top row: 100\% eligibility displays; 80\% eligibility displays; $60 \%$ eligibility displays; $40 \%$ eligibility displays; and $20 \%$ eligibility displays. Left: search for a green T. Right: Search for a red T. 


\section{Three-way ANOVAs}

For target-absent trials, a three-way repeated measures ANOVA with colour, eligibility and display size as factors found a main effect of eligibility $F(1.33,19.94)=71.89, p<.001$, $\eta_{\mathrm{p}}{ }^{2}=.83$ and an interaction between eligibility and display size $F(1.94,29.09)=37.38, p<.001$, $\eta_{\mathrm{p}}{ }^{2}=.71$. Planned contrasts showed that reaction times for displays with $80 \%$ eligibility were already faster than for displays with $100 \%$ eligibility: $71 \mathrm{~ms}, t(15)=2.60, p=.020, d=.65$ and $85 \mathrm{mst}(15)=3.44, p=.004, d=.86$ for display sizes 10 and 20, respectively. When eligibility was reduced further, the reaction time advantage increased, with a larger increase for display size $20(85 \mathrm{~ms}$-> $226 \mathrm{~ms}$-> $461 \mathrm{~ms}$-> $748 \mathrm{~ms})$ than for display size $10(71 \mathrm{~ms}$-> $176 \mathrm{~ms}$-> $319 \mathrm{~ms}->436 \mathrm{~ms})$.

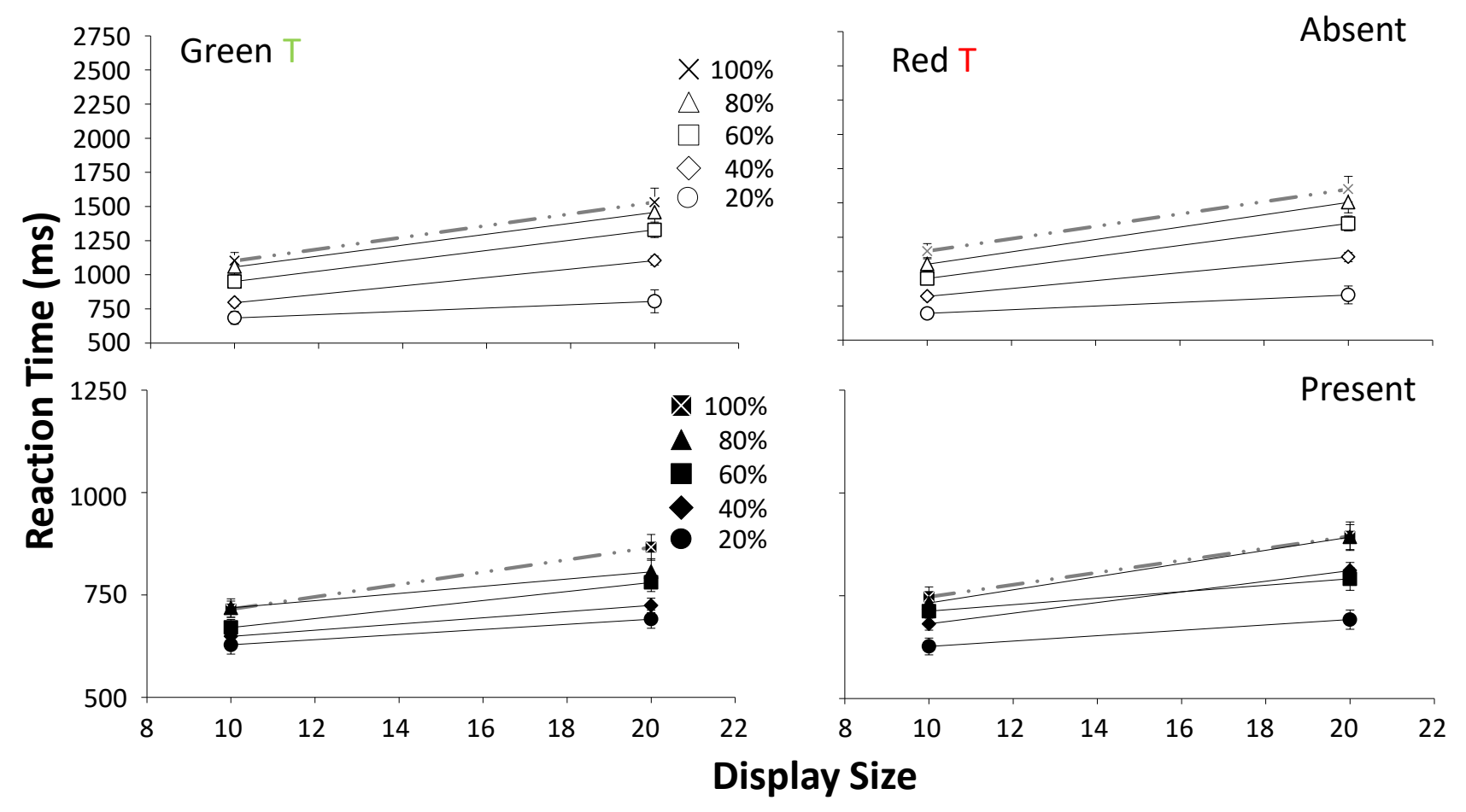

Figure 7. Results of Experiment 3. Reaction times as a function of eligibility, target type, display size and target presence. Left: Green T target; Right: Red T target. Top: absent trials; Bottom: present trials. The grey dashed line is the 100\% eligibility condition. Triangles, squares, diamonds and circles are the $80 \%, 60 \%, 40 \%$, and $20 \%$ eligibility conditions, respectively. The error bars indicate the between-subject standard error of the mean. Slopes can be found in Table A3 in the appendix. 
For target-present trials, a similar three-way ANOVA revealed a three way interaction between colour, eligibility and display size $F(3.21,48.07)=3.40, p=.023, \eta_{\mathrm{p}}{ }^{2}=.19$. The analysis was therefore split further by target colour.

For target-present with a green T, a two-way ANOVA (eligibility x display size) found a significant main effect for eligibility $F(2.99,44.89)=42.73, p<.001, \eta_{\mathrm{p}}{ }^{2}=.74$ and a significant interaction between eligibility and display size $F(3.06,45.89)=3.96, p=.013, \eta_{\mathrm{p}}{ }^{2}=.21$. According to follow up t-tests, eligibility started to confer a reaction time advantage at $80 \%$ for display size 20, [60 ms, $t(15)=2.47, p=.026, d=.62]$ and at $60 \%$ for display size 10 , [45 ms, $t(15)=3.13, p=.007, d=.78]$. For smaller eligibilities, the reaction time advantage further increased, but more so for display size 20. (-3 ms $\rightarrow>45 \mathrm{~ms}->66 \mathrm{~ms} \rightarrow>87 \mathrm{~ms}$ for 10 items vs. $60 \mathrm{~ms}$-> $86 \mathrm{~ms}$-> $142 \mathrm{~ms}$-> $175 \mathrm{~ms}$ for 20 items)

For target-present with a red T, a two-way ANOVA also found a significant main effect for eligibility $F(2.99,44.87)=50.48, p<.001, \eta_{\mathrm{p}}{ }^{2}=.77$ and a significant interaction between eligibility and display size $F(3.26,48.88)=8.74, p<.001, \eta_{\mathrm{p}}{ }^{2}=.37$. Follow up t-test demonstrated that there was a reaction time advantage at $60 \%$ eligibility for both display size 10, [35 ms $t(15)=2.86, p=.012, d=.72]$ and display size $20,[103 \mathrm{~ms} t(15)=5.43, p<.001$, $d=1.36]$. As with the green target, the reaction time advantage increased when eligibility was further reduced, especially for display size 20 , although not without a hiccough at $40 \%$ eligibility. (15 ms -> $35 \mathrm{~ms}$-> $66 \mathrm{~ms}$-> $120 \mathrm{~ms}$ for 10 items vs. $2 \mathrm{~ms}$-> $104 \mathrm{~ms}$-> $83 \mathrm{~ms}$-> $203 \mathrm{~ms}$ for 20 items) 


\section{Errors}

For target-absent trials there were no significant effects eligibility. For target present trials there were more errors for $20 \%$ eligibility than for $100 \%$ eligibility, but only for 10 item displays. Please see the appendix for more detail.

Table 4 Error Rates Experiment 3

\begin{tabular}{|c|c|c|c|c|c|c|c|c|}
\hline & \multicolumn{8}{|l|}{ Colour } \\
\hline & \multicolumn{4}{|l|}{ Green $\mathrm{T}$} & \multicolumn{4}{|l|}{ Red T } \\
\hline & \multicolumn{3}{|l|}{ Target } & & \multicolumn{4}{|l|}{ Target } \\
\hline & \multicolumn{2}{|l|}{ Absent } & \multicolumn{2}{|l|}{ Present } & \multicolumn{2}{|l|}{ Absent } & \multicolumn{2}{|l|}{ Present } \\
\hline & 10 Items & 20 Items & 10 Items & 20 Items & 10 Items & 20 Items & 10 Items & 20 Items \\
\hline Eligibility & & & & & & & & \\
\hline $100 \%$ & .02 & .01 & .02 & .05 & .00 & .02 & .03 & .04 \\
\hline $80 \%$ & .02 & .01 & .02 & .04 & .00 & .00 & .03 & .05 \\
\hline $60 \%$ & .01 & .02 & .03 & .02 & .02 & .01 & .03 & .05 \\
\hline $40 \%$ & .02 & .01 & .03 & .03 & .01 & .01 & .04 & .03 \\
\hline $20 \%$ & .02 & .01 & .05 & .03 & .01 & .01 & .05 & .04 \\
\hline
\end{tabular}

Experiment 4 - Guidance by Orientation

\section{Method}

The target was defined by the orientation of its constituting lines. It was either drawn with lines from the cardinal set (horizontal + vertical; yielding orientations of $-90^{\circ}, 0^{\circ}, 90^{\circ}$, and $180^{\circ}$ ) or lines from the diagonal set (left + right diagonal; yielding orientations of $-135^{\circ},-45^{\circ}$, $45^{\circ}$, and $135^{\circ}$ ). The eligibilities used were $100 \%, 83 \%, 67 \%, 33 \%$ and $17 \%$ and the display size were 12 and 18, with 25 trials per cell of the design. (See Figure 8 for an overview of the stimulus conditions) 


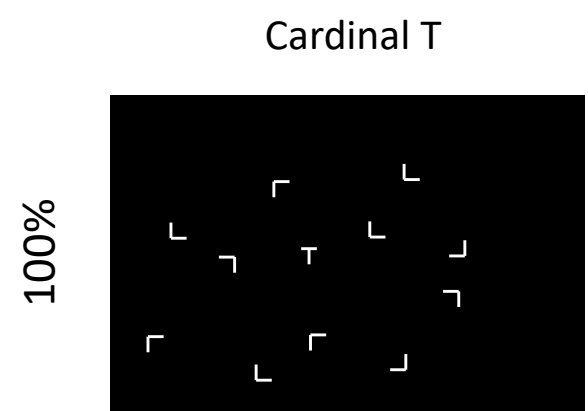

Diagonal $\lambda$
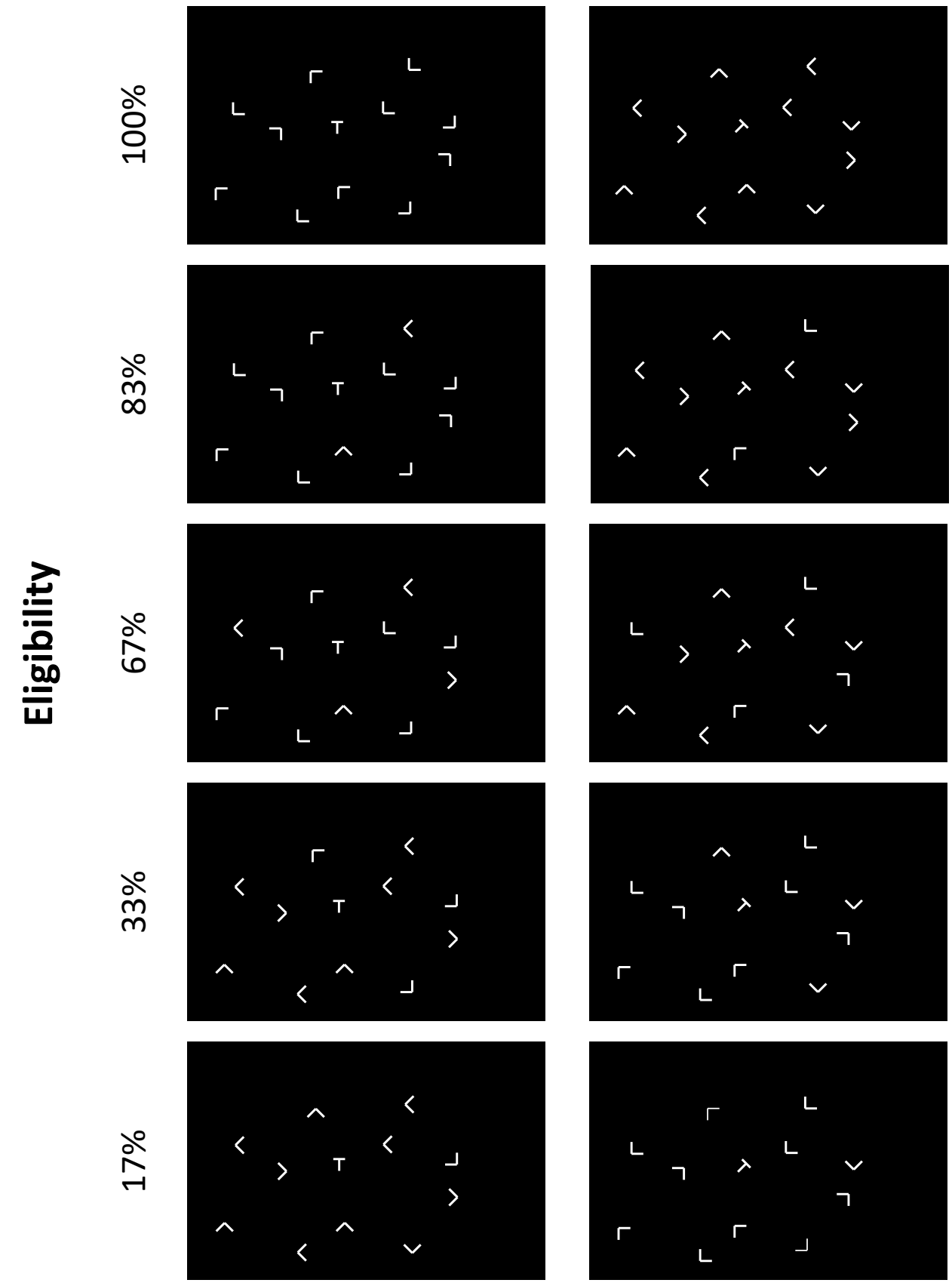

Figure 8. Manipulation used in Experiment 4. From the top row: 100\% eligibility displays; 83\% eligibility displays; $67 \%$ eligibility displays; 33\% eligibility displays; and $17 \%$ eligibility displays. Left: search for a cardinal T. Right: Search for a diagonal T. 
Results

Summary

Performance was very similar, whether the participants searched for a cardinal target or for a diagonal one. For target-absent trials, reaction times increased when the eligibility ratio was reduced to $83 \%$, and even for $17 \%$ eligibility reaction times were still slower than for the displays with $100 \%$ eligibility. For target-present trials, reaction times stayed fairly constant across the eligibility ratios of $83 \%, 67 \%$ and $33 \%$, and the reaction times only improved significantly when eligibility fell to $17 \%$. The errors on target-present trials were reduced for $17 \%$ and $33 \%$ eligibility, but not for the higher eligibility ratios. The reaction times are shown in Figure 9, the error rates are listed in Table 5 and the slopes can be found in Table A4 (appendix). Outlier removal excluded $1.9 \%$ of the trials.

\section{Reaction Times}

\section{Four-way ANOVA}

The four-way ANOVA yielded a significant main effect of eligibility $F(1.96$, 29.38) $=46.45, p<.001, \eta_{\mathrm{p}}{ }^{2}=.76$. There were also significant two-way interactions between eligibility and display size $F(2.47,37.08)=6.04, p=.003, \eta_{\mathrm{p}}{ }^{2}=.29$ and eligibility and target $F(2.24,33.64)=34.23, p<.001, \eta_{\mathrm{p}}^{2}=.70$. As before, the analysis was split along the target dimension and two separate follow-up three-way (eligibility x orientation x display size) ANOVAs were conducted. 


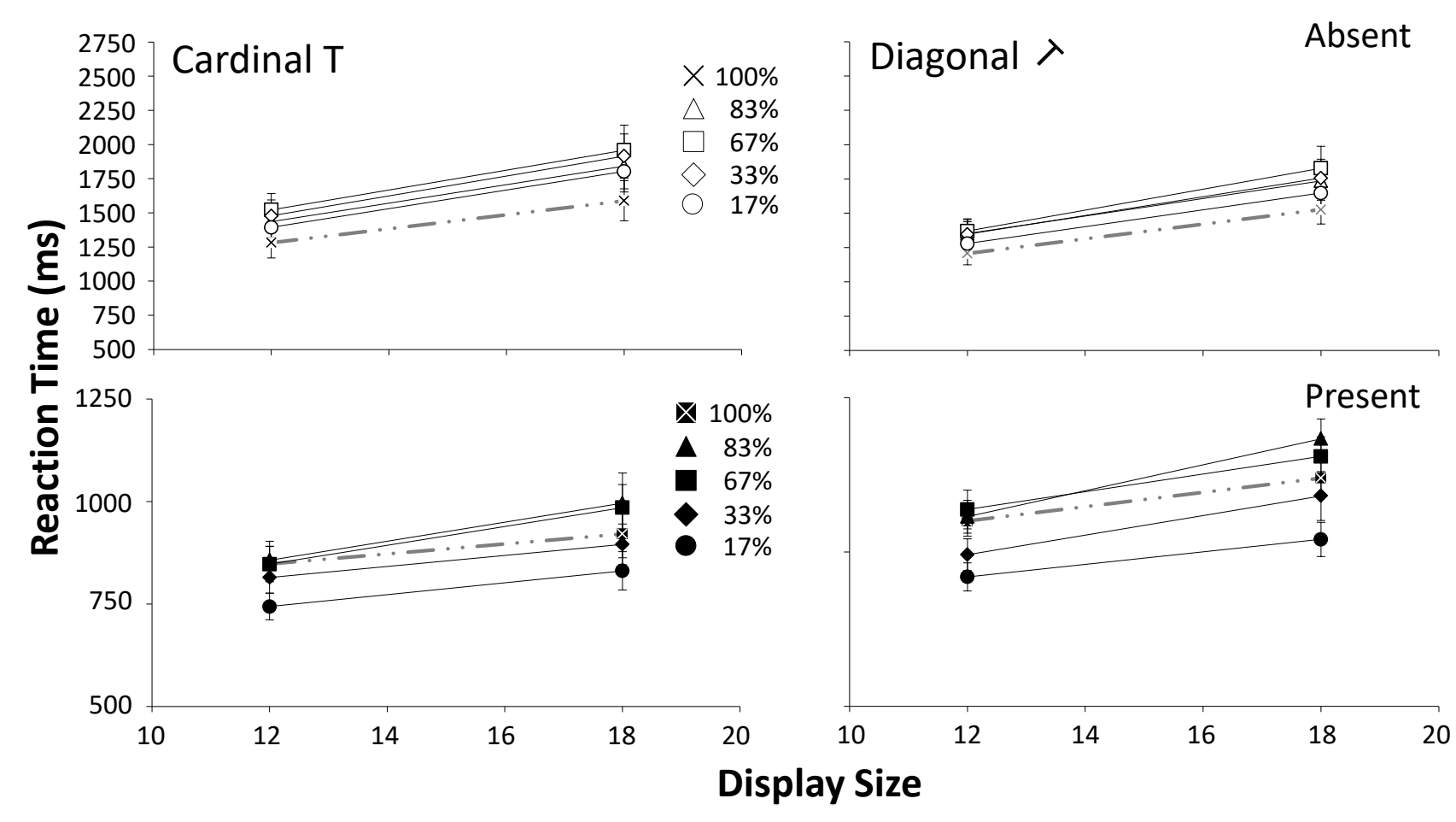

Figure 9. Results of Experiment 4. Reaction times as a function of eligibility, target type, display size and target presence. Left: Cardinal T target; Right: Diagonal T target. Top: absent trials; Bottom: present trials. The grey dashed line is the 100\% eligibility condition. Triangles, squares, diamonds and circles are the $83 \%, 67 \%, 33 \%$, and $17 \%$ eligibility conditions, respectively. The error bars indicate the between-subject standard error of the mean. Slopes can be found in Table A4 in the appendix.

\section{Three-way ANOVAs}

For the target-absent trials, there was no improvement as a function of decreasing eligibility.

In fact, $100 \%$ eligibility had both the fastest reaction times and the shallowest search slopes.

This can be seen from the three-way ANOVA which revealed a significant main effect of eligibility $F(1.70,25.54)=44.81, p<.001, \eta_{\mathrm{p}}{ }^{2}=.75$ and a two-way interaction between eligibility and display size $F(2.55,38.29)=4.78, p=.009, \eta_{\mathrm{p}}{ }^{2}=.24$. Planned contrasts pairing $100 \%$ eligibility with the other levels of eligibility showed that the reaction times in targetabsent trials were fastest for $100 \%$ eligibility: $1401 \mathrm{~ms}$ for $100 \%$ vs. $1591 \mathrm{~ms}, 1670 \mathrm{~ms}, 1624$ $\mathrm{ms}$, and $1531 \mathrm{~ms}$ for $83 \%, 67 \%, 33 \%$, and $17 \%$, respectively (all $p$ 's $<.001, \eta_{\mathrm{p}}{ }^{2}$ 's $>.72$ ).

Similar contrasts also revealed that the search slopes were shallowest for $100 \%$ eligibility 
(comparison of $100 \%$ to other levels: all $p$ ' $\mathrm{s}<.04, \eta_{\mathrm{p}}{ }^{2}$ 's $>.25$, see the appendix for the actual slope values).

For target-present trials, there was only an improvement in reaction times for $17 \%$ eligibility. This follows from a three-way ANOVA that found a significant main effect of eligibility $F(2.40,35.92)=30.26, p<.001, \eta_{\mathrm{p}}{ }^{2}=.67$ and planned contrasts pairing $100 \%$ eligibility with each of the other levels of eligibility. Only the $17 \%$ eligibility level was significantly faster than $100 \%$ (764 ms vs. $860 \mathrm{~ms}), F(1,15)=35.79, p<.001, \eta_{\mathrm{p}}{ }^{2}=.71$. The $83 \%$ and $67 \%$ eligibility levels were numerically slower than 100\% (at $898 \mathrm{~ms}$ and $889 \mathrm{~ms}$, respectively) whereas the $33 \%$ eligibility level was numerically faster (824 ms). Yet, all these contrasts narrowly failed to clear a Bonferroni-corrected critical value of $.0125(.013<$ p's $<.08 ; .20<$ $\left.\eta_{\mathrm{p}}^{2}<.35\right)$

\section{Errors}

There were no effects on absent trials, but for present trials were fewer errors for $17 \%$ and $33 \%$ eligibility. Please see the appendix for more detail.

Table 5 Error Rates Experiment 4

\begin{tabular}{|c|c|c|c|c|c|c|c|c|}
\hline & \multicolumn{8}{|c|}{ Orientation } \\
\hline & \multicolumn{4}{|c|}{ Cardinal T } & \multicolumn{4}{|c|}{ Diagonal T } \\
\hline & \multicolumn{3}{|l|}{ Target } & & \multicolumn{3}{|l|}{ Target } & \\
\hline & \multicolumn{2}{|l|}{ Absent } & \multicolumn{2}{|l|}{ Present } & \multicolumn{2}{|l|}{ Absent } & \multicolumn{2}{|l|}{ Present } \\
\hline & 12 Items & 18 Items & 12 Items & 18 Items & 12 Items & $\overline{18}$ Items & 12 Items & 18 Items \\
\hline Eligibility & & & & & & & & \\
\hline $100 \%$ & .01 & .00 & .06 & .05 & .01 & .00 & .07 & .05 \\
\hline $83 \%$ & .00 & .01 & .05 & .07 & .01 & .01 & .06 & .07 \\
\hline $67 \%$ & .01 & .01 & .05 & .07 & .01 & .00 & .04 & .04 \\
\hline $33 \%$ & .01 & .02 & .02 & .04 & .01 & .02 & .01 & .02 \\
\hline $17 \%$ & .01 & .02 & .01 & .01 & .00 & .01 & .01 & .01 \\
\hline
\end{tabular}


Experiment 5 - Guidance by Motion

\section{Method}

In this experiment the target was defined by its motion status and was either static $0.0 \%$ or moving $7.2 \%$ s. The eligibilities used were $100 \%, 83 \%, 67 \%, 33 \%$ and $17 \%$; the display sizes were 12 and 18 and there were 25 trials per cell of the design. (See Figure 10 for an overview of the stimulus conditions)

Results

Summary

For target-absent trials, there was an immediate improvement in reaction times when the number of eligible items was reduced, but for target-present trials an improvement in reaction times only became manifest when the eligibility ratios were very low at $17 \%$ and $33 \%$. The error proportions remained fairly constant across the levels of eligibility. The reaction times are shown in Figure 11, the error rates are listed in Table 6 and the slopes can be found in Table A5 (appendix). Outlier removal excluded 2.0\% of the trials 
Differences in top-down guiding attributes 36

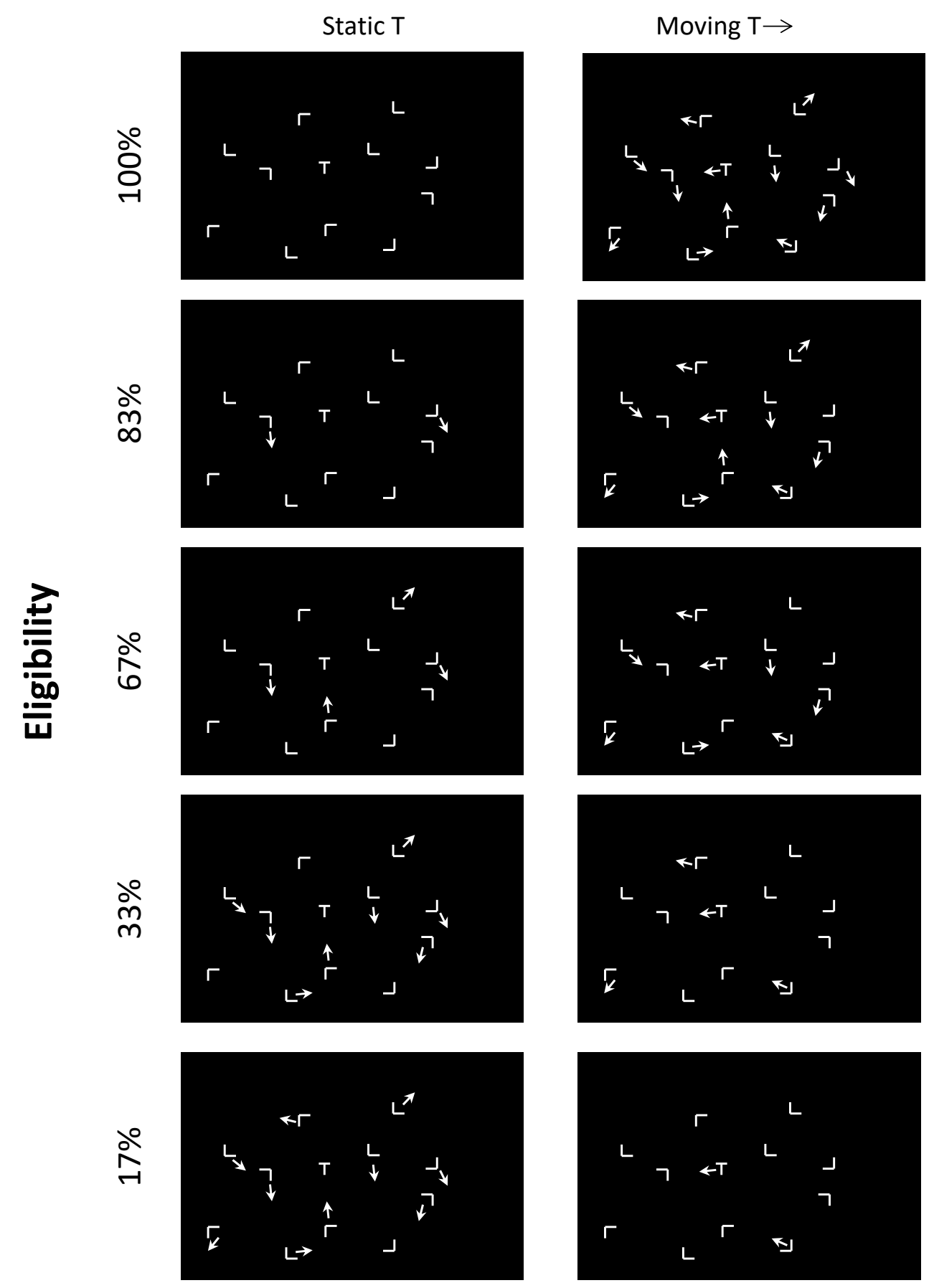

Figure 10. Manipulation used in Experiment 5. From the top row: $100 \%$ eligibility displays; 83\% eligibility displays; 67\% eligibility displays; 33\% eligibility displays; and 17\% eligibility displays. Left: search for a static T. Right: Search for a moving T. 


\section{Reaction Times}

\section{Four-way ANOVA}

The four-way ANOVA showed a significant main effect of eligibility $F(1.16$, 17.47) $=30.58, p<.001, \eta_{\mathrm{p}}{ }^{2}=.67$, significant two-way interactions between eligibility and display size $F(1.96,29.35)=13.49, p<.001, \eta_{\mathrm{p}}{ }^{2}=.47$, eligibility and target $F(1.29$, $19.30)=14.12, p<.001, \eta_{\mathrm{p}}{ }^{2}=.49$ and eligibility and motion $F(1.65,24.78)=6.76, p=.007$, $\eta_{\mathrm{p}}{ }^{2}=.31$, and a three-way interaction between eligibility, display size and target $F(1.68$, $25.12)=4.34, p=.030, \eta_{\mathrm{p}}{ }^{2}=.23$. To further characterise the effect of eligibility, two separate follow-up three-way (eligibility x motion x display size) ANOVAs were conducted on targetabsent and target-present trials.

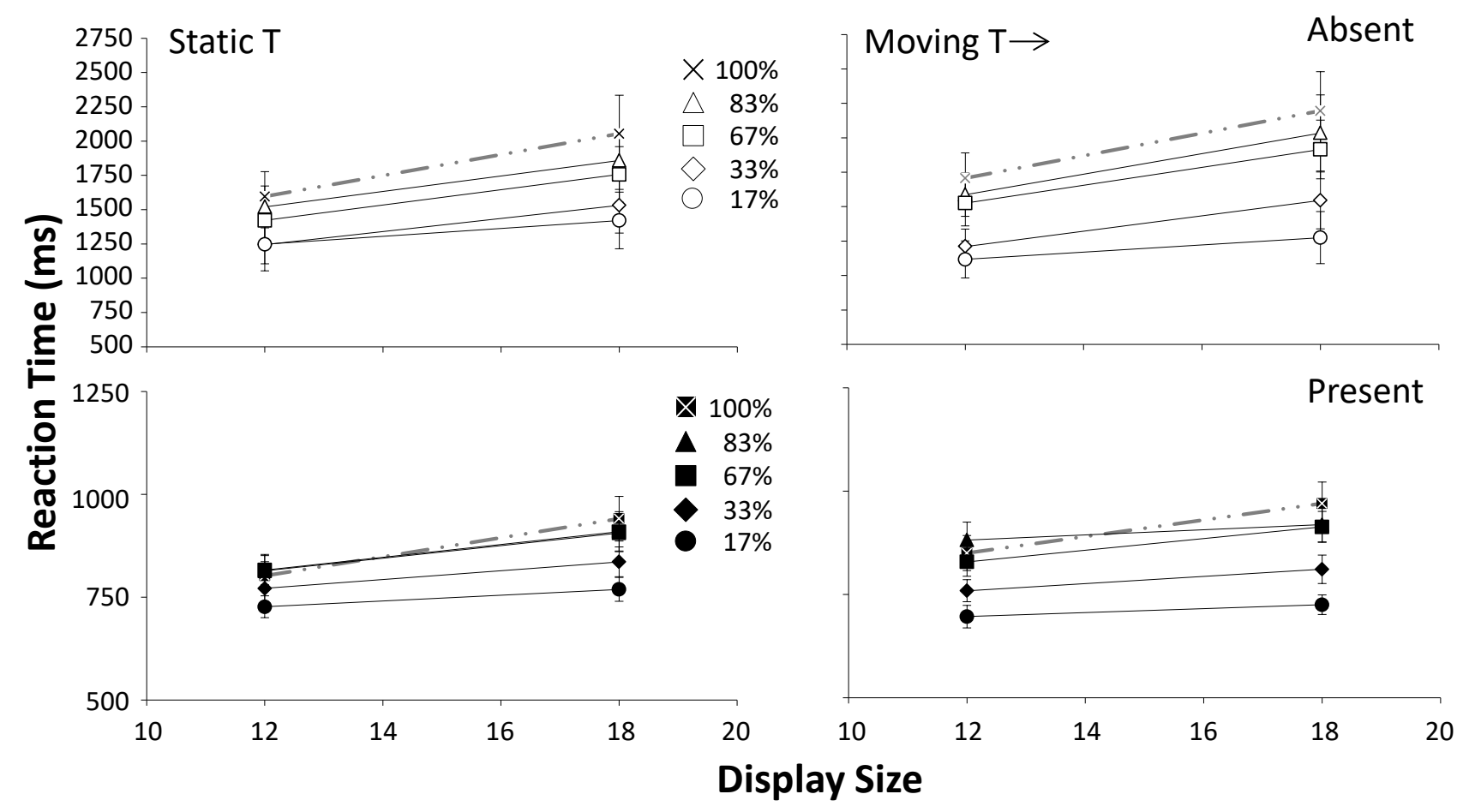

Figure 11. Results of Experiment 5. Reaction times as a function of eligibility, target type, display size and target presence. Left: Static T target; Right: Moving T target. Top: absent trials; Bottom: present trials. The grey dashed line is the 100\% eligibility condition. Triangles, squares, diamonds and circles are the $83 \%, 67 \%, 33 \%$, and $17 \%$ eligibility conditions, respectively. The error bars indicate the between-subject standard error of the mean. Slopes can be found in Table A5 in the appendix. 


\section{Three-way ANOVAs}

For target-absent trials, there was a significant main effect of eligibility $F(1.19$, $17.88)=22.65, p^{<.001,} \eta_{\mathrm{p}}{ }^{2}=.60$. Furthermore, there were significant two-way interactions between eligibility and motion $F(1.57,23.49)=4.90, p=.023, \eta_{\mathrm{p}}{ }^{2}=.25$ and between eligibility and display size $F(1.57,23.55)=9.15, p=.002, \eta_{\mathrm{p}}{ }^{2}=.38$.

The planned contrasts pairing the reaction times of $100 \%$ eligibility with those of the other levels of eligibility revealed that reaction times were already faster for $83 \%$ eligibility, 1750 ms vs. $1888 \mathrm{~ms}, F(1,15)=11.90, p=.004, \eta_{\mathrm{p}}{ }^{2}=.44$ and this advantage continued to grow as eligibility dropped: $1655 \mathrm{~ms}, 1382 \mathrm{~ms}$, and $1265 \mathrm{~ms}$ for $67 \%, 33 \%$ and $17 \%$ respectively. For $17 \%$ eligibility, the reaction time gain was larger for moving targets: $F(1,15)=5.57$, $p=.032, \eta_{\mathrm{p}}^{2}=.27(755 \mathrm{~ms}$ vs. $491 \mathrm{~ms})$. For almost all comparisons with $100 \%$, the reaction time gains were larger for display size 18 than display size 12: 83\% eligibility (178 ms vs, 98 ms) $F(1,15)=9.25, p=.008, \eta_{\mathrm{p}}{ }^{2}=.38 ; 33 \%$ eligibility $(424 \mathrm{~ms}$ vs $288 \mathrm{~ms}) F(1,15)=14.36$, $p=.002, \eta_{\mathrm{p}}{ }^{2}=.49$; and $17 \%$ eligibility $(777 \mathrm{~ms}$ vs. $469 \mathrm{~ms}) F(1,15)=14.15, p=.002, \eta_{\mathrm{p}}{ }^{2}=.49$.

For target-present trials, there was a significant main effect of eligibility $F(2.15$, $32.20)=48.05, p<.001, \eta_{\mathrm{p}}{ }^{2}=.76$, and significant two-way interactions between eligibility and motion $F(2.56,38.46)=4.65, p=.010, \eta_{\mathrm{p}}{ }^{2}=.24$ and between eligibility and display size $F(2.75$, $41.20)=6.56, p=.001, \eta_{\mathrm{p}}{ }^{2}=.30$.

To establish at which levels of eligibility target knowledge yielded a reaction time advantage, a planned contrasts paired $100 \%$ eligibility with each of the other levels of eligibility. The contrasts revealed that the reaction times for $17 \%\left[F(1,15)=72.61, p<.001, \eta_{\mathrm{p}}{ }^{2}=.83\right]$ and $33 \%$ eligibility $\left[F(1,15)=47.92, p<.001, \eta_{\mathrm{p}}{ }^{2}=.76\right]$ were faster than $100 \%(729 \mathrm{~ms}$ and $794 \mathrm{~ms}$ vs. $891 \mathrm{~ms})$. But the contrasts comparing the reaction times of $100 \%$ to those of $67 \%$ and $83 \%$ 
(891 ms vs. $867 \mathrm{~ms}$ and $880 \mathrm{~ms}$ ) failed to clear the Bonferroni-corrected critical value of .01 $\left(\mathrm{p}=.030, \eta_{\mathrm{p}}^{2}=.28\right.$ and $\mathrm{p}=.35, \eta_{\mathrm{p}}^{2}=.06$, respectively).

The reaction time improvements at 17\% were larger for moving targets (199 ms vs. $123 \mathrm{ms,}$ $\left.F(1,15)=8.81, p=.010, \eta_{\mathrm{p}}{ }^{2}=.37\right)$. The reaction time improvements at $17 \%$ and $33 \%$ were also larger for display size 18. [For 33\% eligibility: $132 \mathrm{~ms}$ vs $61 \mathrm{~ms}, F(1,15)=9.02, p=.009$, $\eta_{\mathrm{p}}{ }^{2}=.38$ and for $17 \%$ eligibility: $208 \mathrm{~ms}$ vs $\left.115 \mathrm{~ms}, F(1,15)=15.90, p=.001, \eta_{\mathrm{p}}{ }^{2}=.52\right]$.

\section{Errors}

There were no effects involving eligibility. Please see the appendix for more detail.

Table 6 Error Rates Experiment 5

\begin{tabular}{|c|c|c|c|c|c|c|c|c|}
\hline & \multicolumn{8}{|l|}{ Motion } \\
\hline & \multicolumn{4}{|l|}{ Static T } & \multicolumn{4}{|c|}{ Moving T } \\
\hline & \multicolumn{3}{|l|}{ Target } & & \multicolumn{3}{|l|}{ Target } & \\
\hline & \multicolumn{2}{|l|}{ Absent } & \multicolumn{2}{|l|}{ Present } & \multicolumn{2}{|l|}{ Absent } & \multicolumn{2}{|l|}{ Present } \\
\hline & 12 Items & 18 Items & 12 Items & 18 Items & 12 Items & 18 Items & 12 Items & 18 Items \\
\hline \multicolumn{9}{|l|}{ Eligibility } \\
\hline $100 \%$ & .01 & .00 & .03 & .05 & .00 & .01 & .03 & .06 \\
\hline $83 \%$ & .01 & .01 & .02 & .05 & .00 & .01 & .02 & .04 \\
\hline $67 \%$ & .00 & .00 & .02 & .04 & .01 & .02 & .03 & .02 \\
\hline $33 \%$ & .01 & .00 & .01 & .03 & .01 & .01 & .02 & .02 \\
\hline $17 \%$ & .01 & .00 & .02 & .03 & .01 & .01 & .03 & .03 \\
\hline
\end{tabular}

\section{Discussion of Experiments 3-5}

There is a clear qualitative difference between targets defined by orientation of their constituting lines on the one hand and targets defined by colour or motion of their constituting lines on the other. Whereas there was a steady improvement in search performance when fewer and fewer items had the same colour or motion status as the target, the introduction of items created with ineligibly oriented lines clearly interfered with search performance, especially on absent trials. As discussed in Hulleman et al. (2020), the detrimental effect of orientation in target-present trials may have been balanced out by the presence of the $\mathrm{T}$ which may attract attention in its own right (c.f. Zelinsky, 1996). Probably 
the most remarkable result is the fact that even when the eligible orientation items were surrounded by a sea of ineligible items in $17 \%$ eligibility displays, there still was a performance decrement. This opens up the question whether orientation, rather than a help in the guidance of attention as proposed by Guided Search, might actually be a hindrance ${ }^{5}$.

The final two experiments therefore tested the effects of doubly defining eligibility. In Experiment 6 eligible items had both a unique colour and a unique orientation. In Experiment 7, eligibility was defined by both motion status and orientation. Are guidance by colour and guidance by motion strong enough to withstand the negative effects of orientation? Or, conversely, are colour and motion capable of unlocking the potential of guidance by orientation? The latter is actually what should happen according to Guided Search, since the eligible items should now receive the activation from two of its broadly tuned channels, whereas the ineligible items should not receive any activation at all.

Experiment 6 - Guidance by Colour + Orientation

\section{Method}

In this experiment, the target was defined by a combination of colour and the orientation of its constituting lines: it was either red cardinal or green diagonal. The eligibilities used were $100 \%, 80 \%, 60 \%, 40 \%$ and $20 \%$. The display sizes were 10 and 20 and there were 25 trials per cell of the design. (See Figure 12 for an overview of the stimulus conditions) 


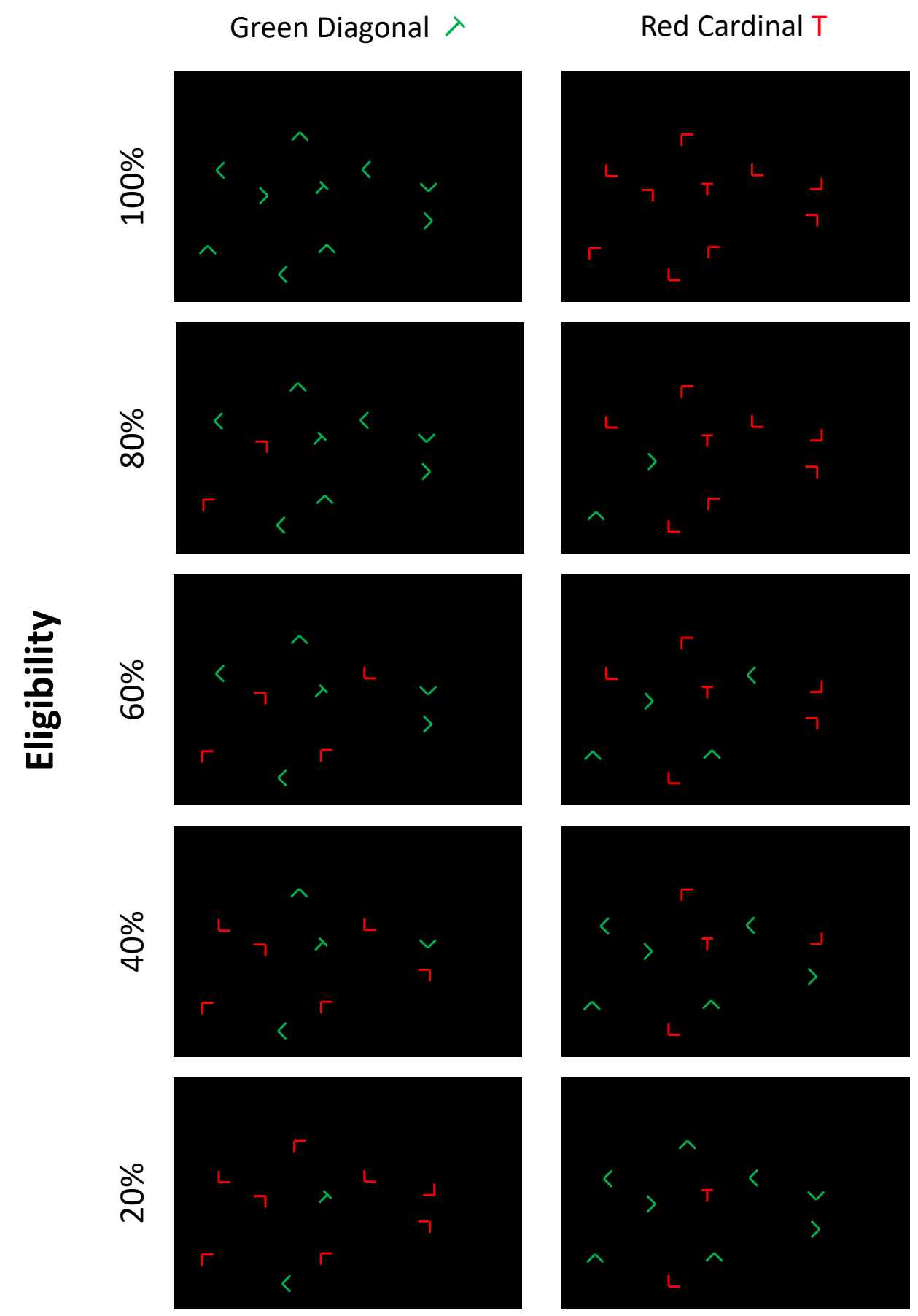

Figure 12. Manipulation used in Experiment 6. From the top row: $100 \%$ eligibility displays; 80\% eligibility displays; $60 \%$ eligibility displays; $40 \%$ eligibility displays; and $20 \%$ eligibility displays. Left: search for a green diagonal T. Right: Search for a red vertical T. 
Results

\section{Summary}

Compared to Experiment 3 the results are fairly similar. It therefore seems safe to conclude that guidance by colour is not impeded by the presence of orientation differences. On the other hand it was also not the case that the addition of orientation improved guidance by colour, something that should have happened according to Guided Search. The reaction times are shown in Figure 13, the error rates are listed in Table 7 and the slopes can be found in Table A6 (appendix). Outlier removal excluded $1.8 \%$ of the trials.

\section{Reaction Times}

\section{Four-way ANOVA}

A four-way repeated measures ANOVA on the reaction times (eligibility x colour $\mathrm{x}$ display size $\mathrm{x}$ target) found a main effect of eligibility $F(1.15,17.22)=126.79, p<.001, \eta_{\mathrm{p}}{ }^{2}=.89$, twoway interactions between eligibility and display size $F(1.75,26.22)=50.41, p<.001, \eta_{\mathrm{p}}{ }^{2}=.77$ and between eligibility and target $F(1.29,19.27)=62.54, p<.001, \eta_{\mathrm{p}}{ }^{2}=.81$ and a three-way interaction between eligibility, display size and target $F(2.07,31.09)=31.76, p<.001, \eta_{\mathrm{p}}{ }^{2}=.68$. Because of these interactions, we split the analysis along the target dimension.

\section{Three-way ANOVAs}

For target-absent trials a three-way repeated measures ANOVA with eligibility, colour and display size as factors found a significant main effect of eligibility $F(1.13,17.00)=105.28$, $p<.001, \eta_{\mathrm{p}}{ }^{2}=.88$ and a significant interaction between eligibility and display size $F(1.34$, 20.08) $=55.91, p<.001, \eta_{\mathrm{p}}{ }^{2}=.79$. Follow-up t-tests revealed that there was already a significant advantage at $80 \%$ eligibility for 10 item displays (68 ms, $t(15)=5.64, p<.001, d=1.41)$ whereas for 20 item display the advantage only became manifest at $60 \%$ eligibility $(267 \mathrm{~ms}$, 
$t(15)=5.81, p<.001, d=1.45)$. Overall, the reaction time advantage due to reduced eligibility increased more steeply for displays with 20 items than those with $10(68 \mathrm{~ms} \rightarrow>171 \mathrm{~ms}$-> $321 \mathrm{~ms}$-> $423 \mathrm{~ms}$ for 10 items and $49 \mathrm{~ms}$-> $267 \mathrm{~ms}$-> $530 \mathrm{~ms}$-> $848 \mathrm{~ms}$ for 20 items).

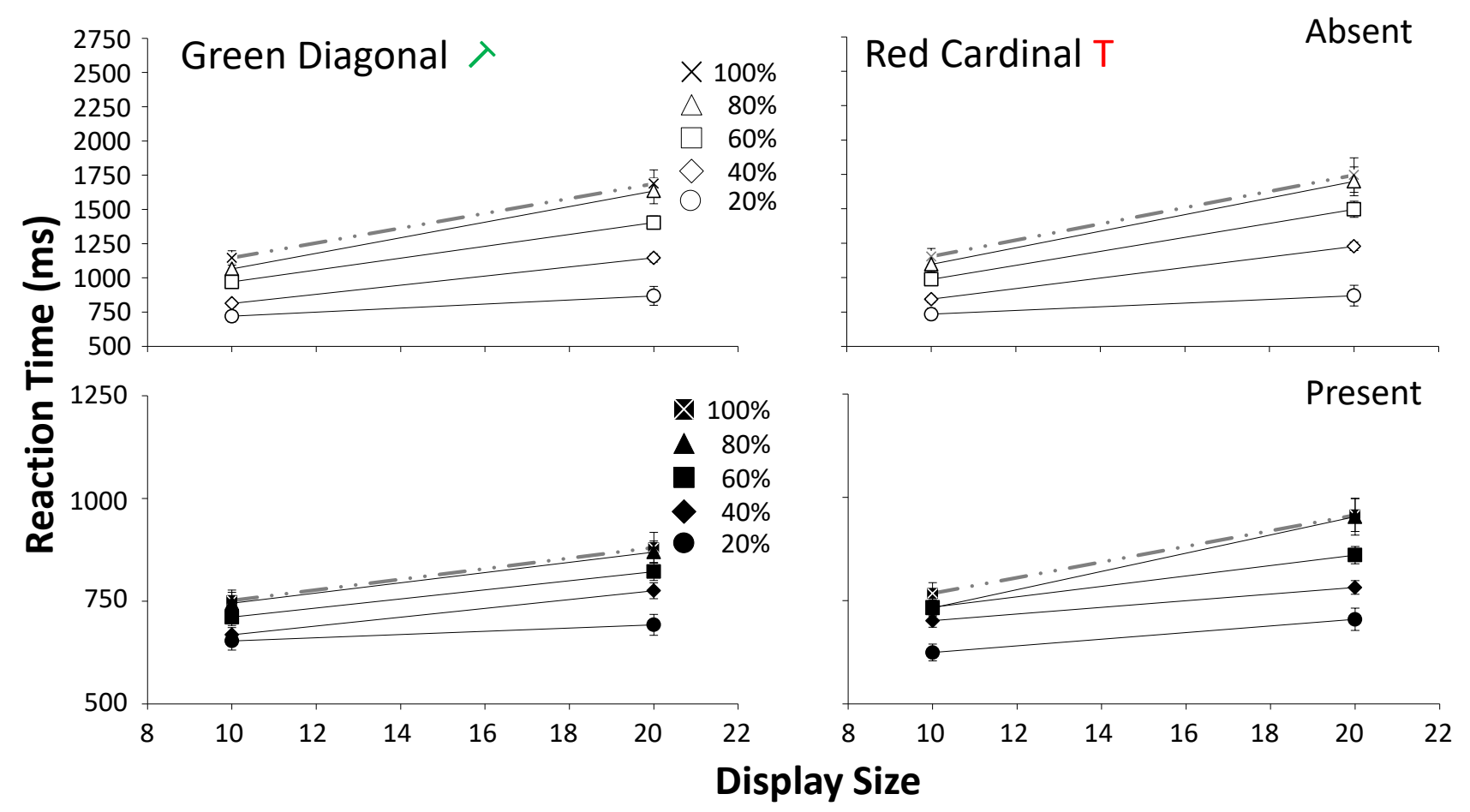

Figure 13. Results of Experiment 6. Reaction times as a function of eligibility, target type, display size and target presence. Left: Green Diagonal T target; Right: Red Cardinal T target. Top: absent trials; Bottom: present trials. The grey dashed line is the 100\% eligibility condition. Triangles, squares, diamonds and circles are the $80 \%, 60 \%, 40 \%$, and $20 \%$ eligibility conditions, respectively. The error bars indicate the between-subject standard error of the mean. Slopes can be found in Table A6 in the appendix.

For target-present trials a similar three-way ANOVA found a significant main effect of eligibility $F(1.77,26.56)=62.54, p<.001, \eta_{\mathrm{p}}^{2}=.81$ and significant two-way interactions between eligibility and display size $F(2.74,41.11)=9.85, p<.001, \eta_{\mathrm{p}}{ }^{2}=.40$ and between colour and display size $F(1,15)=14.28, p=.002, \eta_{\mathrm{p}}{ }^{2}=.49$. The three-way interaction between colour, eligibility and display size came close to significance $F(2.94,44.03)=2.75, p=.055, \eta_{\mathrm{p}}{ }^{2}=.16$. We therefore split our planned comparison against $100 \%$ eligibility both by target colour and by display size. 
For all combinations of colour and display size, the reaction advantage relative to $100 \%$ eligibility increased with decreased eligibility: green 10 items: $7 \mathrm{~ms}$-> $41 \mathrm{~ms}$-> $83 \mathrm{~ms}$-> 98 ms; green 20 items: $11 \mathrm{~ms}$-> $58 \mathrm{~ms}$-> $105 \mathrm{~ms}$-> $188 \mathrm{~ms}$; red 10 items: $35 \mathrm{~ms}$-> $34 \mathrm{~ms}$-> 66 ms -> 143 ms; red 20 items: 4 ms -> 97 ms -> 175 ms -> 252 ms. However the point where the reaction time advantage became manifest varied. For red 10 items it took until $40 \%$ eligibility $t(15)=4.72, p<.001, \mathrm{~d}=1.18$. For the other three combinations of colour and display size there was already a significant advantage at $60 \%$ eligibility: green 10 items $t(15)=2.79$, $p=.014, \mathrm{~d}=.70 ;$ green 20 items $t(15)=2.45, p=.027, \mathrm{~d}=.613 ;$ red 20 item $t(15)=2.77, p=.014$, $\mathrm{d}=.69$.

\section{Errors}

There were no effects involving eligibility. Please see the appendix for more detail.

Table 7 Error Rates Experiment 6

\begin{tabular}{|c|c|c|c|c|c|c|c|c|}
\hline & \multicolumn{8}{|c|}{ Colour + Orientation } \\
\hline & \multicolumn{4}{|c|}{ Green Diagonal T } & \multicolumn{4}{|c|}{ Red Cardinal T } \\
\hline & \multicolumn{3}{|c|}{ Target } & & \multicolumn{3}{|c|}{ Target } & \\
\hline & \multicolumn{2}{|l|}{ Absent } & \multicolumn{2}{|l|}{ Present } & \multicolumn{2}{|l|}{ Absent } & \multicolumn{2}{|l|}{ Present } \\
\hline & 10 Items & 20 Items & 10 Items & 20 Items & 10 Items & 20 Items & 10 Items & 20 Items \\
\hline \multicolumn{9}{|l|}{ Eligibility } \\
\hline $100 \%$ & .03 & .01 & .02 & .05 & .00 & .01 & .04 & .05 \\
\hline $80 \%$ & .02 & .02 & .02 & .03 & .01 & .01 & .03 & .04 \\
\hline $60 \%$ & .03 & .01 & .04 & .03 & .01 & .01 & .03 & .04 \\
\hline $40 \%$ & .02 & .01 & .02 & .03 & .02 & .01 & .03 & .02 \\
\hline $20 \%$ & .01 & .01 & .04 & .03 & .01 & .01 & .04 & .05 \\
\hline
\end{tabular}

Experiment 7 - Guidance by Motion + Orientation

\section{Method}

The target was defined by a combination of motion and the orientation of its constituting lines. The target was either a static diagonal $\mathrm{T}$ or a moving cardinal $\mathrm{T}$. The eligibilities used were $100 \%, 83 \%, 67 \%, 33 \%$ and $17 \%$. The display sizes were 12 and 18 , with 25 trials per cell of the design. (See Figure 14 for an overview of the stimulus conditions) 
Results

\section{Summary}

For both target-absent and target-present trials, reaction times did not improve until the eligibility ratio was reduced to $33 \%$. For target-absent trials this compares unfavourably to Experiment 5 where eligibility was defined by motion status only. It therefore seems that orientation actually interfered with guidance by motion. The reaction times are shown in Figure 15, the error rates are listed in Table 8 and the slopes can be found in Table A7 (appendix). Outlier removal excluded $2.3 \%$ of the trials.

\section{Reaction Times}

\section{Four-way ANOVA}

A four-way repeated measures ANOVA (eligibility $\mathrm{x}$ motion $\mathrm{x}$ display size $\mathrm{x}$ target) yielded a host of effects involving eligibility. There was a main effect of eligibility $F(1.21$, $18.13)=29.91, p<.001, \eta_{\mathrm{p}}{ }^{2}=.67$ showing that reaction times improved when the eligibility ratio was reduced. But this effect of eligibility was qualified by two-way interactions between eligibility and motion $F(1.93,28.95)=23.57, p<.001, \eta_{\mathrm{p}}{ }^{2}=.61$, eligibility and display size $F(3.06,45.88)=13.35, p<.001, \eta_{\mathrm{p}}{ }^{2}=.47$, and eligibility and target $F(1.24,18.58)=12.90$, $p=.001, \eta_{\mathrm{p}}{ }^{2}=.46$ and by three-way interactions between eligibility, motion and target $F(2.03$, $30.45)=14.28, p<.001, \eta_{\mathrm{p}}{ }^{2}=.49$ and between eligibility, display size and target $F(2.78$, $41.75)=6.20, p=.002, \eta_{\mathrm{p}}{ }^{2}=.29$. To characterise the effect of eligibility more fully, two separate follow-up three-way (eligibility x motion x display size) ANOVAs were conducted for targetabsent and target-present trials. 
Differences in top-down guiding attributes 46

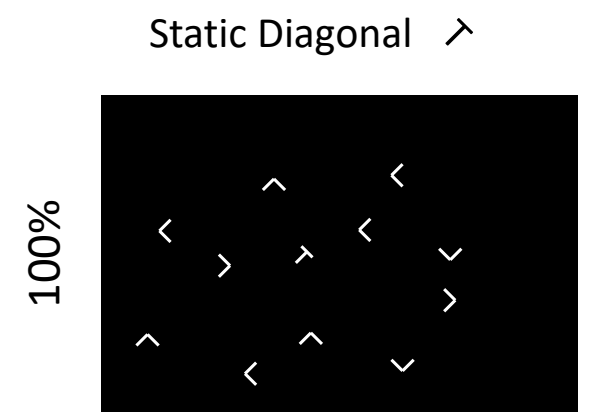

Moving Cardinal $\mathrm{T} \rightarrow$
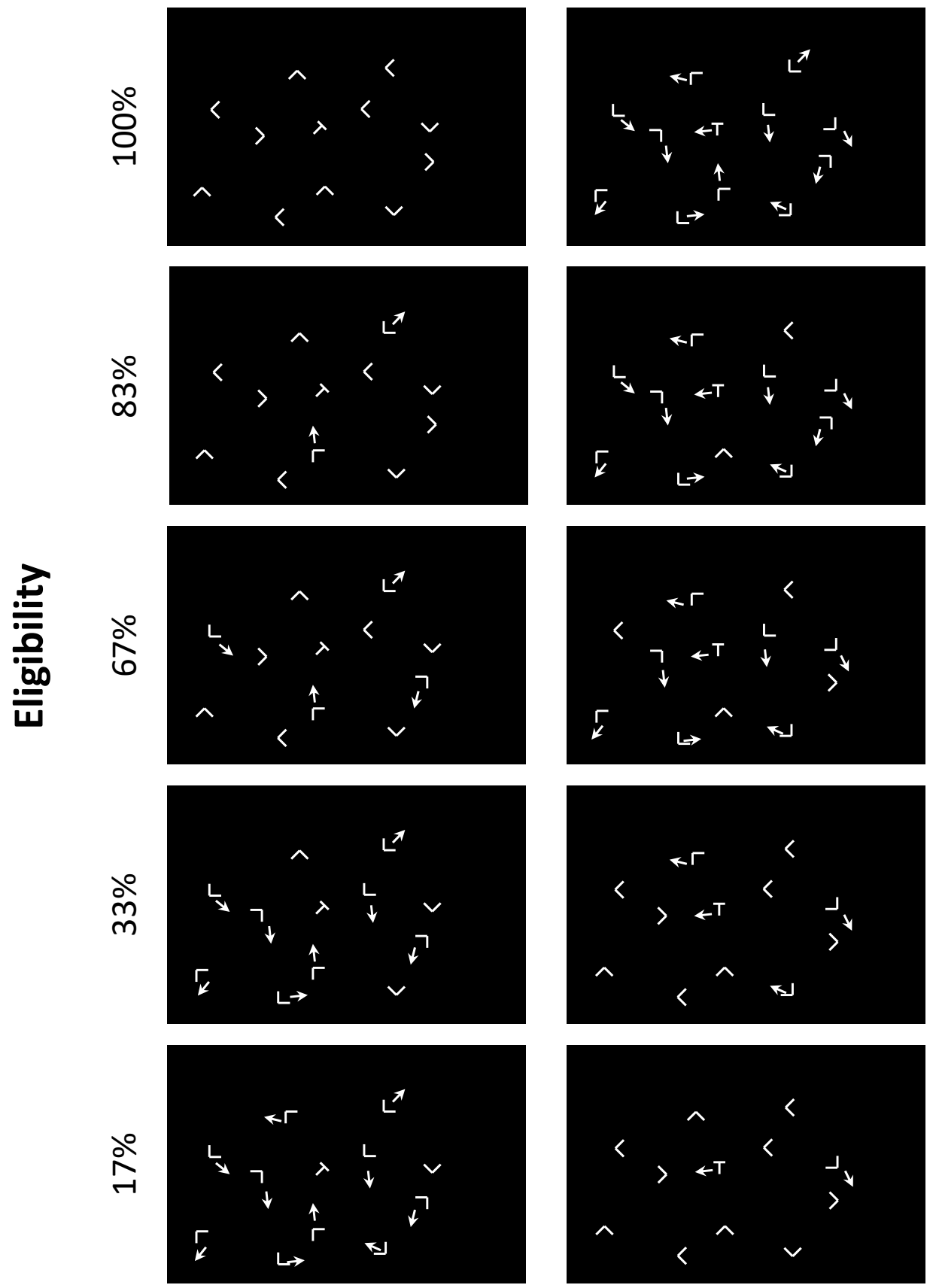

Figure 14. Manipulation used in Experiment 7. From the top row: $100 \%$ eligibility displays; 83\% eligibility displays; $67 \%$ eligibility displays; $33 \%$ eligibility displays; and $17 \%$ eligibility displays. Left: search for a static diagonal T. Right: Search for a moving cardinal $T$. 


\section{Three-way ANOVAs}

For the target-absent trials there was only a reaction time advantage when there were very few eligible items $(33 \%, 17 \%)$. This advantage was larger when participants knew that the target was cardinal and moving, and it was also larger for the larger display size. This follows from the three-way ANOVA which revealed a significant main effect of eligibility $F(1.19$, $17.81)=22.68, p<.001, \eta_{\mathrm{p}}{ }^{2}=.60$ and two-way interactions between eligibility and motion $F(1.82,27.34)=27.35, p<.001, \eta_{\mathrm{p}}{ }^{2}=.65$ and between eligibility and display size $F(2.57$, $38.50)=13.86, p<.001, \eta_{\mathrm{p}}^{2}=.48$.

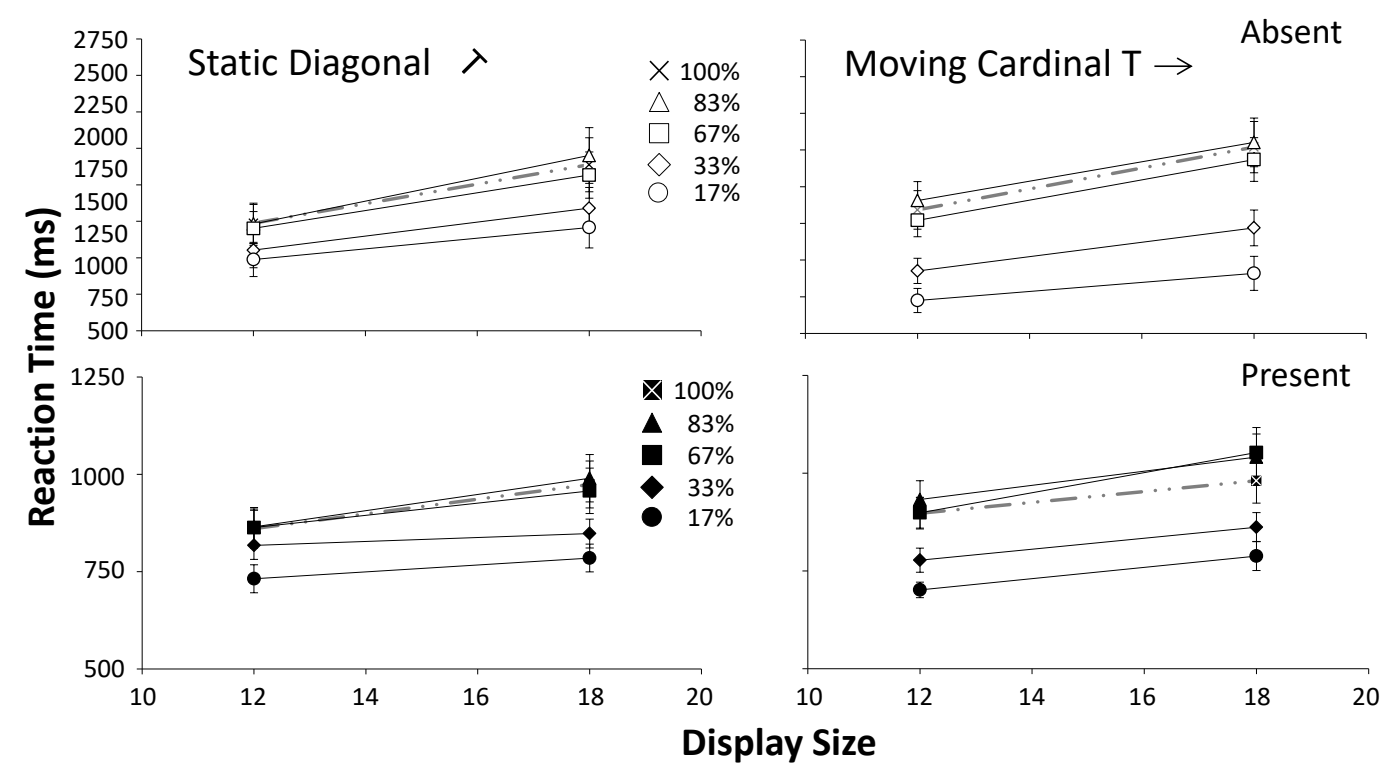

Figure 15. Results of Experiment 7. Reaction times as a function of eligibility, target type, display size and target presence. Left: Static T target; Right: Moving T target. Top: absent trials; Bottom: present trials. The grey dashed line is the 100\% eligibility condition. Triangles, squares, diamonds and circles are the $83 \%, 67 \%, 33 \%$, and $17 \%$ eligibility conditions, respectively. The error bars indicate the between-subject standard error of the mean. Slopes can be found in Table A7 in the appendix.

Planned contrasts on the target absent trials pairing $100 \%$ with the other levels of eligibility revealed that the reaction times for $17 \%$ were faster than for $100 \%, 1208 \mathrm{~ms}$ vs. $1747 \mathrm{~ms}$, $F(1,15)=19.93, p<.001, \eta_{\mathrm{p}}^{2}=.57$. This reaction time improvement was larger for cardinalmoving $F(1,15)=31.59, p<.001, \eta_{\mathrm{p}}^{2}=.68(739 \mathrm{~ms}$ vs. $339 \mathrm{~ms}$ for diagonal-static $)$ and for display size $18 F(1,15)=25.21, p<.001, \eta_{\mathrm{p}}{ }^{2}=.63$ (645 ms vs. $433 \mathrm{~ms}$ for display size 12$)$. The 
same held for 33\% eligibility: faster reaction times than for $100 \%, 1385 \mathrm{~ms}$ vs. $1747 \mathrm{~ms}$, $F(1,15)=13.21, p=.002, \eta_{\mathrm{p}}^{2}=.47$, with a larger reaction time improvement for cardinalmoving $F(1,15)=21.48, p<.001, \eta_{\mathrm{p}}^{2}=.59$ (483 ms vs. 240 for diagonal static) and for display size $18 F(1,15)=20.41, p<.001, \eta_{\mathrm{p}}{ }^{2}=.58$ (424 ms vs. 299 for display size 12$)$. None of the pairwise contrasts comparing $100 \%$ to $83 \%$ and $67 \%$ eligibility was significant (all $p$ 's $>.27$; $\eta_{\mathrm{p}}{ }^{2} \mathrm{~s}<.08$ ), but it should be noted that the reaction times for $83 \%$ were actually numerically larger than for 100\%: $1681 \mathrm{~ms}, 1785 \mathrm{~ms}$, and $1747 \mathrm{~ms}$ for $67 \%, 83 \%$ and $100 \%$ eligibility, respectively.

For target-present trials, the pattern was similar: there was only a reaction time advantage for the lowest levels of eligibility, 33\% and 17\%. For the higher levels eligibility, $67 \%$ and $83 \%$, reaction times did not reliably change relative to the $100 \%$ eligibility level. This can be seen from the outcome of the three-way ANOVA. There was a significant main effect of eligibility $F(1.81,27.21)=42.10, p<.001, \eta_{\mathrm{p}}{ }^{2}=.74$. None of the interactions involving eligibility was significant, although eligibility and motion $F(2.45,36.77)=2.46, p=.09, \eta_{\mathrm{p}}{ }^{2}=.14$ and eligibility and display size $F(2.90,43.46)=2.49, p=.08, \eta_{\mathrm{p}}{ }^{2}=.14$ came close.

Planned contrasts on the target present trials pairing $100 \%$ with each of the other levels of eligibility revealed that only $17 \%$ and $33 \%$ differed reliably from 100\%, $752 \mathrm{~ms}$ and $826 \mathrm{~ms}$ vs. $928 \mathrm{~ms},\left[F(1,15)=50.62, p<.001, \eta_{\mathrm{p}}{ }^{2}=.77 ; F(1,15)=21.19, p<.001, \eta_{\mathrm{p}}{ }^{2}=.59\right.$, respectively $]$. The contrasts comparing $100 \%$ to $83 \%$ and $67 \%$ failed to clear the Bonferroni-corrected critical value of .0125 ( $p$ 's of $.37 ; \eta_{\mathrm{p}}{ }^{2}=.05$ and $.054 ; \eta_{\mathrm{p}}{ }^{2}=.223$, respectively). Please note that for both $83 \%(957 \mathrm{~ms})$ and $67 \%(943 \mathrm{~ms})$ the reaction times were actually numerically slower than for 100\% eligibility (928 ms). 


\section{Errors}

When eligibility was reduced, the difference in error rate between target absent and target present became smaller. Please see the appendix for more detail.

Table 8 Error Rates Experiment 7

\begin{tabular}{|c|c|c|c|c|c|c|c|c|}
\hline & \multicolumn{8}{|c|}{ Motion + Orientation } \\
\hline & \multicolumn{4}{|c|}{ Static Diagonal T } & \multicolumn{4}{|c|}{ Moving Cardinal T } \\
\hline & \multicolumn{3}{|l|}{ Target } & & \multicolumn{3}{|l|}{ Target } & \\
\hline & \multicolumn{2}{|l|}{ Absent } & \multicolumn{2}{|l|}{ Present } & \multicolumn{2}{|l|}{ Absent } & \multicolumn{2}{|l|}{$\overline{\text { Present }}$} \\
\hline & 12 Items & 18 Items & 12 Items & 18 Items & 12 Items & $\overline{18}$ Items & 12 Items & $\overline{18}$ Items \\
\hline Eligibility & & & & & & & & \\
\hline $100 \%$ & .02 & .02 & .04 & .02 & .01 & .01 & .03 & .04 \\
\hline $83 \%$ & .01 & .00 & .05 & .05 & .01 & .00 & .03 & .03 \\
\hline $67 \%$ & .00 & .02 & .03 & .02 & .01 & .02 & .02 & .02 \\
\hline $33 \%$ & .01 & .01 & .02 & .01 & .01 & .01 & .03 & .03 \\
\hline $17 \%$ & .02 & .02 & .01 & .02 & .01 & .02 & .03 & .02 \\
\hline
\end{tabular}

\section{Discussion of Experiments 6-7}

The final two experiments revealed some subtle differences between motion and colour as a guiding attribute. Orientation seemed to act as a drag at higher levels of eligibility when added to motion status: whereas in Experiment 5 (motion) reaction times became faster when eligibility was reduced to $83 \%$, reaction times in Experiment 7 (motion + orientation) actually became numerically slower. This impression of a negative effect of the addition of orientation to the target definition is supported for both absent and present trials by significant interaction contrasts that compared the reaction times for $100 \%$ and $83 \%$ eligibility in Experiment 5 and Experiment 7: $F(1,30)=11.35, p=.002, \eta_{\mathrm{p}}{ }^{2}=.27$ and $F(1$, 30) $=4.97, p=.033, \eta_{\mathrm{p}}{ }^{2}=.14$ for target-absent and present, respectively.

The addition of orientation to colour did not seem to have any negative effects on the effectiveness of attentional guidance. Reaction times became faster when eligibility was reduced to $80 \%$ irrespective of whether target was defined by colour (Experiment 3 ) or colour and orientation (Experiment 6 ). Indeed, the $100 \%$ vs $80 \%$ contrasts for a comparison of Experiment 3 and Experiment 6 yielded no significant results: $F(1,30)=.38, p=.54, \eta_{\mathrm{p}}{ }^{2}=.012$ 
and $F(1,30)=.11, p=.74, \eta_{\mathrm{p}}{ }^{2}=.004$ for absent and present trials. In that sense colour is a stronger guiding attribute than motion, since it is more impervious to the effects of orientation. What did not happen should also be noted. Doubling the definition of eligibility by using both orientation and motion/colour did not lead to superior performance relative to the situation where eligibility was only defined by either motion or colour. Contrary to the predictions of Guided Search ${ }^{6}$, at no point did direct comparisons between Experiments 3 and 5 on the one hand and Experiments 6 and 7 on the other yield a significant effect that indicates that doubly defined items yield better guidance.

\section{General Discussion}

These results paint a more complicated picture than one would be led to assume by the list of "undoubted" guiding attributes in Wolfe and Horowitz (2004; 2017). Whereas reducing eligibility by changing the colour (Experiments 2 and 3) or the motion status (Experiments 1 and 4) of distractors improved search performance, changing their orientation impeded it (Experiment 4). Even when eligibility fell to $17 \%$, performance in target-absent trials was still not better than in $100 \%$ eligibility trials where all items had the same orientation. So, rather than merely being weaker, orientation seems to reside in a fundamentally different class from motion and colour. The differences between motion and colour are more nuanced, but Experiments 6 and 7 suggest that guidance by colour is more robust than guidance by motion $^{7}$.

Some readers may wonder whether these experiments compare like with like, given that the colour of the T was always identical when it was defined by colour whereas its orientation varied when it was defined by the orientation of the line set. Could this variability have made 
the orientation information less useful? Although this suggestion may sound plausible, it actually does not hold for the following two reasons.

First, it is important to make a distinction between the orientation of the $\mathrm{T}$ and the orientation of the individual component lines of the $\mathrm{T}$. The individual component lines of the $\mathrm{T}$ ( $\mathrm{a}$ horizontal and vertical line for the cardinal condition and left and right tilted lines for the diagonal condition) were always identical. What changed from trial to trial was their configuration. As a consequence, the orientation of the T did indeed vary across trials. But according to the assumptions underlying both FIT and Guided Search, it is the orientation of the constituent lines (which remained constant for all trials in the diagonal and cardinal conditions) rather than the orientation of the resulting configuration that provides the guidance of attention. Second, Hulleman et al. (2020) actually did use a target T where the configuration of the lines was kept constant (i.e. the T always had the same orientation). Nevertheless, they too found that adding distractors with ineligible orientations reduced search performance rather than improving it. This was even the case in a 5 vs 2 experiment were the target was an upright 5 with an aspect ratio of 1:2 and the ineligible orientation distractors were 2's that were all tilted 45 degrees clockwise. Consequently, it seems that it is not the varying orientation of the $\mathrm{T}$ that is responsible for the poor performance of orientation as a guiding attribute in the current experiments but rather the poor top-down guidance by orientation per se.

A related concern may be that the orientation of the individual lines was lost when they were combined into T and L's. However, Hulleman et al. (2020) showed that search performance for a particular configuration of two squares improved when half of the distractors were made 
ineligible by rotating them 45 degrees. This suggests that the orientation of constituting lines is not necessarily lost when they become part of a configuration.

It may seem curious that this paper finds clear qualitative and quantitative differences between attributes that have been grouped together as "undoubted guiding attributes" by Wolfe and Horowitz (2004, 2017). But it is important to note here that the wealth of evidence for this status provided by Wolfe and Horowitz $(2004,2017)$ is of a very particular kind. Mostly, it concerns experiments where the target has a unique attribute value (e.g. red amongst green, / amongst |, moving amongst static). Usually, these experiments yield flat search slopes. These flat search slopes are seen as evidence for guiding status of the unique attribute (much in the same as they would have been evidence for feature status three decades ago). But in terms of Guided Search it is actually unclear whether this guidance is top-down or bottom-up: Participants may be able to find the target quickly because it is diagonal, or because there is a clear orientation contrast between the target and its surrounding distractors. The current experiments represent a test of the relative strength of the top-down and bottomup signal that is provided by the various guiding attributes. What the experiments show is that while colour, motion and orientation may all be very effective in terms of bottom-up guidance (as demonstrated by flat search slopes in singleton experiments where the target has a unique colour, motion or orientation), they differ markedly in terms of their capability to provide top-down guidance. Colour stands out as the attribute that is equally effective in bottom-up guidance and in top-down guidance. Whether in singleton experiments or in configuration search with ineligible items, guidance by colour improves search performance markedly. Motion, although a little bit less strongly, also provides top-down and bottom-up guidance. But orientation only seems to provide bottom-up guidance. The current results show that any top-down guidance by orientation is not nearly strong enough to keep in check 
the bottom-up signal created by the orientation differences between eligible and ineligible items.

Some might argue that a conclusion that orientation mainly provides bottom-up guidance is too strong and point at results for conjunction searches that use orientation but not colour as evidence for top-down guidance by orientation. For instance, Sagi (1988) found that a target that is the overlap of two Gabor patches (low frequency horizontal + high frequency vertical) is easily found between distractors that are the overlap of the complementary Gabor patches (low frequency vertical + high frequency horizontal). He concluded that a combination of spatial frequency and orientation is effortlessly perceived. Yet, it should be noted that he did not find effortless search for single Gabor patches. Neither for a low frequency vertical patch amongst high frequency vertical and low frequency horizontal patches, nor for a high frequency horizontal patch amongst high frequency vertical and low frequency horizontal patches. An important factor here is that in the two Gabor patches task all distractors are identical, whereas in the one Gabor tasks there are two kinds of distractors. Consequently, it may be the case that rather than top-down guidance by a combination of orientation and spatial frequency, the effortless search in the two Gabor patches task is the result of the removal of the strong bottom-up orientation signals that are present in the single Gabor patch tasks. Wolfe, Yee and Friedman-Hill (1992) is another study that has been interpreted as evidence for top-down guidance by orientation. Wolfe et al. (1992) found that search for conjunction of curvature and orientation yielded target-present slopes of about $4.8 \mathrm{~ms} /$ item. Again, this could be taken as evidence that there is strong guidance by the combination of curvature and orientation. However, the target-absent slopes of about $20.5 \mathrm{~ms} /$ item suggest that any top-down guidance starts to falter when there is no target. 
So, it seems that neither Sagi (1988) nor Wolfe et al. (1992) provides unqualified support for the notion of strong top-down guidance by orientation. Taking the current results, which do provide a strong test of top-down guidance of by orientation, together with other studies where top-down guidance by orientation seems to have failed (or at the very least seems to have underwhelmed): Bilsky and Wolfe (1995); Hulleman et al. (2020); Poisson and Wilkinson (1992); Olds et al. (2009); Treisman (2006), it does not seem an overly strong conclusion to suggest that any top-down guidance by orientation is easily overpowered by bottom-up signals.

That there might be qualitative differences between colour and other guiding attributes actually also becomes clear from a closer reading of the VWM-literature. For instance, in Luck and Vogel (1997) performance with orientation is never quite up to the level of colour (although this never gets tested formally) and in Maljkovic and Nakayama (1994) there too is a hint that priming effects might not be as strong with spatial frequency as they are with colour. Even stronger evidence about disparities between holding colour and other guiding attributes in VWM comes from Soto et al. (2006) who report: "although shape matching alone was ineffective in guiding attention" (p.1014). Yet, seizing on a marginally significant interaction between guidance by colour and guidance by a conjunction of colour and shape ( $\mathrm{p}=.03)$, the sentence continues: "there was evidence for conjunction information from both the color and the shape of the stimuli playing a role in top-down attentional guidance" (p.1014). In fact, so entrenched seems to be the conviction that what is found for colour is representative for other guiding attributes that the authors then go on to conclude: "In our paradigm, a strong signal may be generated when the color and shape of an item in the search array matches the WM stimulus, overriding even the salient search target" (p.1018). So, even though Soto et al. (2006) did not find evidence that shape provides any guidance on 
its own, they still use the guidance they found for colour to conclude that colour and shape generate a strong signal.

The current results do of course not mean that conclusions based on work with colour are incorrect. But they do suggest that what is found with colour might not be found at all with other guiding attributes. In that sense it is important to realise that rather than colour showing the strongest guiding effects, these guiding effects might actually be unique to colour and its conjunctions.

If we do accept that colour is the strongest top-down guiding attribute that is preferentially used by participants, then a reinterpretation of some classic search results becomes necessary. One of the most influential experiments in the history of visual search was conducted by Wolfe, Cave and Franzel (1989), since it laid the foundation for Guided Search. Their experiment had four conditions where participants searched for a triple conjunction. In the first condition the target was a big red O amongst big green X's, small red X's and small green O's. So, each of the three types of distractors shared one attribute with the target. In the second condition, the small red $\mathrm{X}$ was the target and the big red $\mathrm{O}$ took over its role as distractor. Again, each of the three distractors shared one attribute with the target. In the third condition, the distractors shared two attributes with target. Here, participants searched for a big red O amongst big red X's, big green O's and small red O's. The final condition was simple conjunction search for a big red O amongst big red X's and big green O's. Slopes for the first condition were almost flat $(0.5$ and $1.2 \mathrm{~ms} /$ item for target present and absent respectively) and slowly increased through the second and third condition until they were 5.2 and $10.0 \mathrm{~ms} /$ item for the conjunction search of the fourth condition. These results have usually been interpreted as a clear vindication of the Guided Search Model. Conditions 1 and 2 yield the best search performance, because the target activation receives input from all three attribute channels, and the distractors only from one each. Condition 3 is slower, because 
here the distractors get input from two attribute channels. Consequently, the target stands out less and is actually not different from the simple conjunction search of Condition 4.

However, if we focus on the conditions where there is a big red $\mathrm{O}$ target, it seems that there may be a different explanation for the slope increase from Condition 1 to Condition 4 . This has to do with the number of big red X's in the display. This increases from none in Condition 1 via about 1/3 of the items in Condition 3 to about $1 / 2$ in Condition 4 . Under this interpretation, it is not the number of shared attributes that is the driving force, but rather the effective number of distractors that accompany the big red $\mathrm{O}$ target. Filtering by colour gets rid of all green distractors and because the bigger items will be brighter when luminance is not controlled, only the big red X's remain. This means that what looks like a manipulation of the number of attributes shared may actually be a manipulation of effective set size that is dominated by the colour of the items.

Manipulation of the number of items with the same colour as the target seems also a viable explanation for another result that has been interpreted as evidence for Guided Search. Nordfang and Wolfe (2014) reported a steady increase in search slopes when distractors shared more attributes with a target that was a conjunction of six attributes (colour, orientation, and shape of a smaller shape superimposed on a larger shape). Again it should be noted that if shapes are only allowed to share a single attribute, only $1 / 6$ of the distractors will have a large shape with the same colour as the target. If, on the other hand, distractors are allowed to share five attributes, only $1 / 6$ of them will not have a large shape with the same colour as the target. So, again, what may look like a manipulation of the number of attributes shared, could actually be seen as a proxy for manipulating the number of distractors that share their colour with the target. In fact, an unreported experiment (Wolfe, personal communication) seems to confirm this analysis. In this control experiment, all distractors shared three attributes with the target, but $50 \%$ of the distractors always had the same colour 
as the target. If the number of distractors that share the target colour is the actual factor that determines the search slopes, rather than the number of shared attributes in general, this manipulation should yield slopes that are comparable to the share three attributes condition of the original experiment (since here as well, $50 \%$ of the distractors share their colour with the target). The observed search slopes in the control experiment were $7.3 \mathrm{~ms} /$ item for targetpresent and $28 \mathrm{~ms} /$ item for target-absent. This is indeed right between the slopes for the share two and share three attributes conditions in the original experiment and therefore consistent with a major role for colour in determining search slopes.

The idea that colour dominates visual search receives further support from a paper that appeared just before this manuscript was submitted for the first time. Alexander, Nahvi and Zelinsky (2019) reported several experiments that tested the effect of a mismatch between the orientation of a preview of a photo-realistic target and the orientation of the target in the actual search display. Alexander et al. (2019) found that changing the orientation had no effect in colour displays, but did have an effect in grey scale displays. So, when participants could rely on colour, they were not hindered by changes to the orientation in the target. Only when colour was no longer available for guidance did an effect of orientation change emerge. The current results and those of Alexander et al. (2019) both complement and strengthen each other. Alexander et al. (2019) used a task where the target was always present, whereas this paper used a present/absent task. This paper used a T vs L task, but Alexander et al. (2019) used search for photo-realistic objects. Alexander et al. (2019) found that participants like to rely on colour (in an echo of the results of Poisson \& Wilkinson, 1992) and only use orientation when they have to. The experiments reported here provide a reason for this reliance on colour: orientation provides very poor top-down guidance. Taken together, the papers demonstrate that orientation provides poor top-down guidance in a variety of tasks, thereby undercutting the suggestion that the lack of guidance by orientation observed is 
somehow due to the particular task and/or stimuli used. It is probably also instructive to think about the important difference between Alexander et al. (2019) and the current experiments. In the final analysis, Alexander et al. (2019) did find evidence of guidance by orientation, whereas we did not. This may be a consequence of the difference in difficulty of the task used. When search was very difficult and every item had to be inspected individually, Hulleman et al. (2020) also found evidence of guidance by orientation. Individual inspection of items also seems to have been necessary in the grey-scale stimuli used by Alexander et al. (2019). For instance, despite the fact that participants had to fixate the target during response for a trial to be counted as correct, accuracy was $95.8 \%$ or better for all experiments.

The finding that colour plays a very important role in visual search would probably not come as a surprise to researchers from other fields. For instance, in psycho-linguistics the phenomenon of referential overspecification is well-known. The colour of the target object in an array of distractor object is included in its description, even when colour is not necessary to identify the object (Koolen, Gatt, Goudbeek, Krahmer, 2011; Koolen, Goudbeek, Krahmer, 2013; Pechmann, 1989). For example, in an experiment where participants had to describe one of two objects, Belke and Meyer (2002) found that participants used the colour of the target object redundantly up to $80.2 \%$ of the time (e.g. saying "black ball" to distinguish a black ball from a white star) when their task was to ensure that a listener could identify it in a display (meaning that "ball" would have been enough to accomplish this).

It also seems logical that colour should have a prominent role in visual search. What gets tested in the lab is a capability that is frequently needed in the real world. As already pointed out in Bilsky and Wolfe $(1995)^{8}$, colour is more invariant than orientation. This makes it more reliable as a cue to find the target. When you are looking for a green book on a cluttered desk, its orientation is unpredictable, but its colour will be unchanged. As reported by Wolfe et al. (1994) colour is even useful for multi-coloured objects as long as one colour 
predominates. This is probably a reflection of the real world: even when objects may have patterns, there is quite often one dominating colour. One obvious exception are stripes and it is probably no coincidence that Wally of "Where's Wally" fame is wearing a jumper that has red and white horizontal stripes. This makes finding Wally the tribulation that it is, since it knocks out the most powerful weapon in our visual search arsenal.

The current results raise the question where the performance advantage of colour (and to a lesser extent motion) arises. One suggestion was already made by Egeth et al. (1984): figureground segregation. In a way, this suggests that items with an ineligible colour never make it to the stage where they are available for attentional selection. Note that the figure-ground segregation stage is not an explicit part of Guided Search ${ }^{9}$. According to Wolfe (1994): “At some point, independent parallel representations are generated for a limited set of basic features." (p.204). It is implicitly assumed that these are the right features, i.e. the features that belong to the search items, rather than to the background. If red and green items were presented on a blue background, then there would only be activity in the red and green channels, not in the blue channel. This means that there is a critical difference between a Guided Search and a figure-ground segregation account of search for red vertical amongst red horizontal and green vertical. According to Guided Search, there is activity in both the red and the green colour channel, and it is the combination of activation from the red colour channel and the vertical orientation channel that provides the top-down guidance. But according to a figure-ground segregation account, there is only activity in the red colour channel, since the green is considered background. Under a figure-ground segregation account then, the red vertical target would subsequently be found via the bottom-up activation that the orientation contrast between the red target and the red distractors generates. 


\section{Conclusion}

The strength of colour as a guiding attribute becomes abundantly clear from the experiments reported in this paper ${ }^{10}$. It is by far the best in helping participants reduce set size by separating eligible from ineligible items. In fact, it seems that it is in a category of its own. At the other extreme we find orientation: introducing ineligibly oriented items actually hinders participants, rather than helping them. Somewhere in between we find motion. It helps, but not as well as colour and it is also less impervious to the influence of orientation. What we have to conclude then is that the category of "undoubted guiding attributes" as defined by Wolfe and Horowitz (2017) is a bit of a misnomer. The members of this category are both quantitatively and qualitatively different. The current experiments demonstrate that it is unwise to group colour, orientation and motion together. This also means that it is misleading to take performance with colour as representative for all other guiding attributes. The field's love affair with the manipulation of colour is understandable (due to the strength of the effects obtained), but it is also dangerous because there is no guarantee that performance will generalise to other guiding attributes. 


\section{References}

Alexander, R. G., Nahvi, R., J., \& Zelinsky, G. J. (2019) Specifying the precision of guiding features for visual search. Journal of Experimental Psychology: Human Perception and Performance. doi:10.1037/xhp0000668.

Anderson, G.M., Heinke, D., \& Humphreys, G.W. (2010). Featural guidance in conjunction search: the contrast between orientation and color. Journal of Experimental Psychology: Human Perception \& Performance, 36, 1108-1127. doi: $10.1037 / \mathrm{a} 0017179$

Beck, V.M., Hollingworth, A., \& Luck, S.J. (2012). Simultaneous control of attention by multiple working memory representations. Psychological Science, 23, 887-898. doi: $10.1177 / 0956797612439068$

Belke, E. \& Meyer, A. S. (2002) Tracking the time course of multidimensional stimulus discrimination: Analyses of viewing patterns and processing times during "same"“different” decisions. European Journal of Cognitive Psychology, 14, 237-266, doi: $10.1080 / 09541440143000050$

Bilsky, A. B. \& Wolfe, J. M. (1995). Part-whole information is useful in visual search for size $\mathrm{x}$ size but not orientation $\mathrm{x}$ orientation conjunctions. Perception \& Psychophysics, 57, 749-760. doi: 10.3758/BF03206791

Carrasco, M. \& Yeshurun, Y. (1998). The contribution of covert attention to the set-size and eccentricity effects in visual search. Journal of Experimental Psychology: Human Perception \& Performance, 24, 673-692. doi: 10.1037/0096-1523.24.2.673

Duncan, J., \& Humphreys, G. W. (1989). Visual search and stimulus similarity. Psychological Review, 96, 433-458. doi: 10.1037/0033-295X.96.3.433 
D’Zmura, M. (1991). Color in visual search. Vision Research, 31, 951-966. doi: $10.1016 / 0042-6989(91) 90203-\mathrm{H}$

Egeth, H. E., Virzi, R. A., \& Garbart, H. (1984). Searching for conjunctively defined targets. Journal of Experimental Psychology: Human Perception \& Performance, 10, 32-39. doi: 10.1037/0096-1523.10.1.32

Faul, F., Erdfelder, E, Buchner, A., \& Lang, A. G. (2009). Statistical power analyses using G*Power 3.1: Tests for correlation and regression analyses. Behaviour Research Methods, 41, 1149-1160. doi: 10.3758/BRM.41.4.1149

Hulleman, J., Lund, K., \& Skarratt, P.A. (2020). Medium versus difficult visual search: How a quantitative change in the functional visual field leads to a qualitative difference in performance. Attention, Perception \& Psychophysics, 82, 118-139. doi: 10.3758/s13414-019-01787-4

Hulleman, J. \& Olivers, C. N. L. (2017a). The impending demise of the item in visual search. Behavioral and Brain Sciences, 40, 1-20. doi: 10.1017/S0140525X15002794

Hulleman, J. \& Olivers, C. N. L. (2017b). On the brink: The demise of the item in visual search moves closer. Behavioral and Brain Sciences, 40, 49-69. doi: $10.1017 / \mathrm{S} 0140525 \mathrm{X} 16000364$

Koolen, R., Goudbeek, M., \& Krahmer, E. (2013). The effect of scene variation on the redundant use of color in definite reference. Cognitive Science, 37, 395-411. doi: $10.1111 / \operatorname{cogs} .12019$

Kristjansson, A., Wang, D., \& Nakayama, K. (2002). The role of priming in conjunctive visual search. Cognition, 85, 37-52. doi: 10.1016/S0010-0277(02)00074-4 
Luck, S.J., \& Vogel, E.K. (1997). The capacity of visual working memory for features and conjunctions. Nature, 390, 279-281. doi: 10.1038/36846

Maljkovic, V. \& Nakayama, K. (1994). Priming of pop-out: 1. Role of features. Memory \& Cognition, 22, 657-672. doi: 10.3758/BF03209251

Nordfang, M., \& Wolfe, J.M. (2014). Guided search for triple conjunctions. Attention, Perception \& Psychophysics, 76, 1535-1559. doi: 10.3758/s13414-014-0715-2

Olds, E. S., Graham, T. J., \& Jones, J. A. (2009). Feature head-start: conjunction search following progressive feature disclosure. Vision Research, 49, 1428 1447. doi: 10.1016/j.visres.2009.02.008

Palmer, J., Verghese, P. \& Pavel, M. (2000) The psychophysics of visual search. Vision Research, 40, 1227-1268. doi: 10.1016/S0042-6989(99)00244-8.

Pechmann, T. (1989). Incremental speech production and referential overspecification. Linguistics, 27, 89-110. doi: 10.1515/ling.1989.27.1.89

Poisson, M. E., \& Wilkinson, F. (1992). Distractor ratio and grouping processes in visual conjunction search. Perception, 21, 21-38. doi: 10.1068/p210021

Sobel, K.V., \& Cave, K.R. (2002). Roles of saliency and strategy in visual search. Journal of Experimental Psychology: Human Perception \& Performance, 28, 1055-1070. doi: 10.1037/0096-1523.28.5.1055

Soto, D., Heinke, D., Humphreys, G.W., \& Blanco, M.J. (2005). Early, involuntary top-down guidance of attention from working memory. Journal of Experimental Psychology: Human Perception and Performance, 31, 248-261. doi: 10.1037/0096-1523.31.2.248 
Soto, D., Humphreys, G.W., Heinke, D. (2006). Working memory can guide pop-out search. Vision Research, 46, 1010-1018. doi: 10.1016/j.visres.2005.09.008

Treisman, A. (2006). How the deployment of attention determines what we see. Visual Cognition, 14, 411-443. doi: 10.1080/13506280500195250

Treisman, A. M., \& Gelade, G. (1980). A feature-integration theory of attention. Cognitive Psychology, 12, 97-136. doi: 10.1016/0010-0285(80)90005-5

Van Moorselaar, D., Theeuwes, J. \& Olivers, C.N.L. (2014). In competition for the attentional template: can multiple items within visual working memory guide attention. Journal of Experimental Psychology: Human Perception and Performance, 40, 14501464. doi: $10.1037 / \mathrm{a} 0036229$

Wolfe, J. M. (1994). Guided Search 2.0: A revised model of visual search. Psychonomic Bulletin \& Review, 1, 202-238. doi: 10.3758/BF03200774

Wolfe, J. M. (2007). Guided Search 4.0: Current Progress with a model of visual search. In W. D. Gray (Ed.), Integrated Models of Cognitive Systems (pp. 99-119). New York: Oxford University Press.

Wolfe, J. M. (2018). Visual Search. In J. T. Serences \& J. T. Wixted (Eds.), Stevens' Handbook of Experimental Psychology and Cognitive Neuroscience $4^{\text {th }}$ Edition Volume 2: Sensation, Perception, \& Attention (pp.569-624). Hoboken, NJ: John Wiley \& Sons. doi: 10.1002/9781119170174.epcn213

Wolfe, J. M., Cave, K. R., \& Franzel, S. L. (1989). Guided Search: an alternative to the feature integration model for visual search. Journal of Experimental Psychology: Human Perception \& Performance, 15, 419-433. doi: 10.1037/0096-1523.15.3.419 
Wolfe, J. M., Friedman-Hill, S. R., \& Bilsky, A. B. (1994). Parallel processing of part-whole information in visual search tasks. Perception \& Psychophysics, 55, 537-550. doi: 10.3758/BF03205311

Wolfe, J.M., \& Horowitz, T.S. (2004). What attributes guide the deployment of visual attention and how do they do it? Nature Reviews Neuroscience, 5, 495-501. doi: $10.1038 / \mathrm{nrn} 1411$

Wolfe, J.M., \& Horowitz, T.S. (2017). Five factors that guide attention in visual search. Nature Human Behaviour, 1, 0058, 1-8. doi: 10.1038/s41562-017-0058

Wolfe, J. M., Yee, A., \& Friedman-Hill, S. R. (1992). Curvature is a basic feature for visual search tasks. Perception, 21, 465-480. doi: 10.1068/p210465

Zelinsky, G.J. (1996). Using eye saccades to assess the selectivity of search movements. Vision Research, 36, 2177-2187. doi: 10.1016/0042-6989(95)00300-2

Zhang, W, \& Luck, S.J (2008). Discrete fixed -resolution representations in visual working memory. Nature, 453, 233-235. doi:10.1038/nature0686 


\section{Footnotes}

${ }^{1}$ Although this independence might not hold indefinitely, it has been observed in displays with 36 items (e.g. Carrasco \& Yeshurun, 1998). Most visual search experiments do not venture very far beyond display sizes of 20 items.

${ }^{2}$ Please note that this paper focuses on the qualitative and quantitative differences between the "undoubted" guiding attributes as listed in Wolfe and Horowitz $(2004,2017)$. In that sense, this is less of an investigation into the various theories of visual search and more an investigation into the assumption shared by these theories that any differences between the guiding attributes are purely a matter of degree, not kind.

The list of guiding attributes is most closely associated with Guided Search and FIT. But there are of course alternatives to these two theories, probably most prominently the Attentional Engagement Theory (AET) of Duncan and Humphreys $(1989,1992)$. However, Hulleman et al. (2020) goes into extensive detail about how AET makes the same predictions (albeit for different reasons) as Guided Search and FIT for the eligibility manipulation used in this paper. So, from that point of view, a focus on FIT and Guided Search does not entail any loss of generality.

It should also be noted that FIT and Guided Search are the clearest about which guiding attributes they consider as primitives. Duncan \& Humphreys $(1989,1992)$ are much more circumspect when they speak about matching an input against a target template, although they do mention motion, colour, size and shape. Again, it seems that these attributes are listed in no particular order, implying that they are qualitatively similar. (Please see the work of Heinke \& Humphreys, 2003 and Abadi, Yahya, Amini, Friston \& Heinke, 2019 for a computational implementation of AET's principles). 
Consequently, this paper will use FIT and Guided Search as its theoretical framework.

${ }^{3}$ Given the results of Egeth et al. (1984), Poisson and Wilkinson (1992), Sobel and Cave (2002) and Anderson et al. (2010) it seems unlikely that performance will deteriorate when half of the items in T vs L search is given a different colour. However, as pointed out, this possibility cannot be completely excluded.

${ }^{4}$ Please note that no attempt was made to ensure isoluminance. In all likehood there were clear differences in luminance between the red (RGB 255, 0, 0) and green (RGB 0, 255, 0) items used in these experiments.

${ }^{5}$ Some readers might point out that Anderson et al. (2010) actually did find a positive effect of the orientation cue in their experiments, whereas here we found a negative effect of orientation knowledge. There are at least two points in order here. First, Anderson et al. (2010) only analysed target-present trials. As becomes clear from both the current experiments and Hulleman et al. (2020), the clearest negative effects of orientation are observed in target-absent trials. Second, it might be the case that the orientation cue was most helpful in the verification stage, rather than in the guidance stage (Alexander, Nahvi \& Zelinsky, 2019). The search target in Anderson et al. (2010) could either be blue horizontal or green vertical. Knowing the orientation of the target makes it is easier to establish whether it has been found.

\footnotetext{
${ }^{6}$ Better performance for doubly defined targets is also predicted by AET, because there will be a larger mismatch between the target template that contains both eligible attributes and the ineligible items that contain neither.
}

\footnotetext{
${ }^{7}$ Perhaps these differences between colour, orientation and motion are less surprising then they seem. As noted by one of the anonymous reviewers: Given these biological/neurological
} 
differences in how the features are represented, in hindsight, it seems incredibly unlikely that they would end up having equal influence on perceptual tasks of any type (including visual search).

${ }^{8}$ And independently by one of the anonymous reviewers

${ }^{9}$ Neither does it seem to be an explicit part of AET. It uses "a parallel, hierarchically structured representation of input across the visual field" (Duncan \& Humphreys, 1989, p.445). There is also description of what this representation may contain, but this more closely resembles a list of desirable properties rather than a clear specification of how to derive it.

${ }^{10}$ Please note that the differences between eligible and ineligible items used in the current experiments were categorical (red vs. green; cardinal vs. diagonal; static vs. moving). This was necessary to allow the most sensitive test of the strength of the various guiding attributes. It makes the fact that there was no evidence for top-down guidance by orientation all the more compelling. But it is of course the case that the guiding strength of colour can be attenuated by reducing the linear separability of the colours that define eligible and ineligible items (c.f. D'Zmura, 1991). In that sense, this paper has only demonstrated that the maximum guiding strength of colour is higher than that of motion and orientation. 


\section{Appendix}

\section{Experiment 1 - Motion}

\section{Error Analysis}

The error rates were analysed with a four-way (eligibility $\mathrm{x}$ motion $\mathrm{x}$ display size $\mathrm{x}$ target) repeated measures ANOVA. It found a significant three-way interaction between eligibility, motion and target $F(1,15)=7.01, p=.018, \eta_{\mathrm{p}}{ }^{2}=.32$. Separate eligibility $\mathrm{x}$ motion $\mathrm{x}$ display size ANOVAs for target-absent and target-present revealed that there were no effects whatsoever on the target-absent trials (all $F^{\prime}$ 's $(1,15)<2.88$, all $p$ 's $>$.11, all $\left.\eta_{\mathrm{p}}{ }^{2}{ }^{\prime}<<.17\right)$. For target-present trials however, there was a two-way interaction between eligibility and motion $F(1,15)=6.60, p=.021, \eta_{\mathrm{p}}{ }^{2}=.31$. For static targets the error rate did not depend on eligibility (.023 vs .019 for $100 \%$ and 50\% eligibility, respectively), but for moving targets the error rates increased when eligibility fell (from .014 for $100 \%$ eligibility to .038 for $50 \%$ eligibility). None of the other effects was significant (all $F$ 's $(1,15)<2.07$, all $p$ 's $>.17$, all $\left.\eta_{\mathrm{p}}^{2}{ }^{2}<.13\right)$.

Table A1 Slopes (ms/item) Experiment 1

\begin{tabular}{|c|c|c|c|c|}
\hline & \multicolumn{4}{|l|}{ Motion } \\
\hline & \multicolumn{2}{|l|}{ Static T } & \multicolumn{2}{|c|}{ Moving T } \\
\hline & Absent & Present & Absent & Present \\
\hline Eligibility & & & & \\
\hline $100 \%$ & 67 & 27 & 83 & 18 \\
\hline $50 \%$ & 61 & 16 & 61 & 18 \\
\hline
\end{tabular}

Experiment 2 - Colour

Error Analysis

A four-way repeated measures ANOVA (eligibility $\mathrm{x}$ colour $\mathrm{x}$ display size $\mathrm{x}$ target) on the errors unveiled significant two-way interactions between colour and eligibility $F(2.49$, 
$37.31)=3.22, p=.042, \eta_{\mathrm{p}}{ }^{2}=.18$ and between eligibility and display size $F(2.79,41.88)=3.51$, $p=.026, \eta_{\mathrm{p}}{ }^{2}=.19$ and a three-way interaction between eligibility, display size and target $F(2.27,34.05)=6.25, p=.004, \eta_{\mathrm{p}}^{2}=.29$. As before, we split the analysis by target.

For target-absent trials there were no significant effects in a three-way repeated measures ANOVA (colour x eligibility x display size): all $p$ 's $>.16\left(\eta_{\mathrm{p}}{ }^{2}{ }^{\prime}{ }^{<} \cdot 13\right)$ with the exception of the main effect of colour $F(1,15)=3.29, p=.09, \eta_{\mathrm{p}}^{2}=.18$.

For target-present trials the three-way repeated measures ANOVA found a significant interaction between eligibility and display size $F(2.40,35.98)=5.58, p=.005, \eta_{\mathrm{p}}{ }^{2}=.27$. For 12 item displays the error rates were $.037, .022, .040$, and .045 for $100 \%, 75 \%, 50 \%$ and $25 \%$ eligibility, respectively. For 20 item displays the error rates were $.055, .048, .022$, and .038 . Follow-up $t$-tests revealed the interaction was due to 20 item condition, where $50 \%$ eligibility had a better error rate than $100 \%$ eligibility $t(15)=2.32, p=.035, \mathrm{~d}=.58$.

Table A2 Slopes (ms/item) Experiment 2

\begin{tabular}{|c|c|c|c|c|}
\hline & \multicolumn{4}{|l|}{ Colour } \\
\hline & \multicolumn{2}{|l|}{ Green $\mathrm{T}$} & \multicolumn{2}{|l|}{ Red T } \\
\hline & Absent & Present & Absent & Present \\
\hline \multicolumn{5}{|l|}{ Eligibility } \\
\hline $100 \%$ & 77 & 17 & 77 & 23 \\
\hline $75 \%$ & 72 & 10 & 71 & 25 \\
\hline $50 \%$ & 58 & 19 & 60 & 13 \\
\hline $25 \%$ & 30 & 5 & 27 & 8 \\
\hline
\end{tabular}


Experiment 3 - Colour

\section{Error Analysis}

The four-way repeated measures ANOVA on the error proportions found a significant four way interaction between colour, eligibility, display size and target $F(2.74,41.15)=3.00$, $p=.045, \eta_{\mathrm{p}}{ }^{2}=.17$. As before, we therefore split the analysis along the target dimension.

For target-absent trials there were no significant effects in a three-way colour $\mathrm{x}$ eligibility $\mathrm{x}$ display size ANOVA, although the main effect of colour came close $F(1,15)=4.10, p=.06$, $\eta_{\mathrm{p}}{ }^{2}=.22$ with slightly more error for green T's than for red T's (.014 vs. .009). All other $p$ 's were larger than $.14\left(\eta_{\mathrm{p}}{ }^{2} \mathrm{~s}<.14\right)$.

For target-present trials a similar three-way ANOVA found a significant interaction between eligibility and display size $F(3.15,47.22)=2.85, p=.045, \eta_{\mathrm{p}}{ }^{2}=.16$. Follow-up t-tests revealed that this interaction was due to a significant difference in errors between $100 \%$ eligibility and 20\% eligibility for 10 item displays: .020 vs $.052, t(15)=2.67, p=.017, d=.67$.

Table A3 Slopes (ms/item) Experiment 3

\begin{tabular}{|c|c|c|c|c|}
\hline & \multicolumn{4}{|l|}{ Colour } \\
\hline & \multicolumn{2}{|l|}{ Green $\mathrm{T}$} & \multicolumn{2}{|l|}{ Red T } \\
\hline & Absent & Present & Absent & Present \\
\hline \multicolumn{5}{|l|}{ Eligibility } \\
\hline $100 \%$ & 43 & 15 & 45 & 15 \\
\hline $80 \%$ & 40 & 9 & 45 & 16 \\
\hline $60 \%$ & 38 & 11 & 40 & 8 \\
\hline $40 \%$ & 31 & 8 & 29 & 13 \\
\hline $20 \%$ & 12 & 6 & 13 & 7 \\
\hline
\end{tabular}


Experiment 4 - Orientation

Error Analysis

A four-way repeated measures ANOVA yielded significant main effects of eligibility $F(2.43,36.40)=9.76, p<.001, \eta_{\mathrm{p}}{ }^{2}=.40$ and a significant interaction between eligibility and target $F(2.09,31.35)=9.74, p<.001, \eta_{\mathrm{p}}^{2}=.39$. (All other $p$ 's involving eligibility $>.11$; $\left.\eta_{\mathrm{p}}^{2}{ }^{2}<.13\right)$.

Planned comparisons pairing $100 \%$ with the other levels of eligibility found that for $17 \%$ and $33 \%$ eligibility there were fewer errors $\left(p ' s<.006, \eta_{\mathrm{p}}{ }^{2}>.40\right)$. But the interaction between eligibility and target indicates that this was due to a reduction in errors on the target-present trials, rather than on the target-absent trials.

Table A4 Slopes (ms/item) Experiment 4

\begin{tabular}{|c|c|c|c|c|}
\hline & \multicolumn{4}{|c|}{ Orientation } \\
\hline & \multicolumn{2}{|c|}{ Cardinal T } & \multicolumn{2}{|c|}{ Diagonal T } \\
\hline & Absent & Present & Absent & Present \\
\hline \multicolumn{5}{|l|}{ Eligibility } \\
\hline $100 \%$ & 51 & 12 & 53 & 12 \\
\hline $83 \%$ & 68 & 23 & 64 & 21 \\
\hline $67 \%$ & 73 & 23 & 76 & 14 \\
\hline $33 \%$ & 73 & 13 & 68 & 16 \\
\hline $17 \%$ & 68 & 15 & 61 & 10 \\
\hline
\end{tabular}


Experiment 5 - Motion

Error Analysis

A four-way repeated measures ANOVA (eligibility x motion $\mathrm{x}$ display size $\mathrm{x}$ target) on the errors did not yield any effects involving eligibility. Of all effects involving eligibility, the interaction between eligibility and target came closest: $F(2.34,35.10)=3.00, p=.055$, $\eta_{\mathrm{p}}{ }^{2}=.17$ (all other $\left.p^{\prime} \mathrm{s}>.15 ; \eta_{\mathrm{p}}{ }^{2}{ }^{\mathrm{s}}<.11\right)$. The only effect that did reach significance was that of target $F(1,15)=19.38, p<.001, \eta_{\mathrm{p}}^{2}=.56$, with more errors on target-present trials.

Table A5 Slopes (ms/item) Experiment 5

\begin{tabular}{|c|c|c|c|c|}
\hline & \multicolumn{4}{|l|}{ Motion } \\
\hline & \multicolumn{2}{|l|}{ Static T } & \multicolumn{2}{|c|}{ Moving T } \\
\hline & Absent & Present & Absent & Present \\
\hline \multicolumn{5}{|l|}{ Eligibility } \\
\hline $100 \%$ & 76 & 23 & 82 & 20 \\
\hline $83 \%$ & 56 & 15 & 75 & 6 \\
\hline $67 \%$ & 56 & 16 & 65 & 14 \\
\hline $33 \%$ & 48 & 11 & 56 & 9 \\
\hline $17 \%$ & 29 & 7 & 26 & 5 \\
\hline
\end{tabular}

Experiment 6 - Colour + Orientation

Error Analysis

A four-way repeated measures ANOVA on the error proportions with eligibility, colour, display size and target as factor did not find any significant effects involving eligibility or colour, although the interaction between colour and target came close $F(1,15)=3.87, p=.07$, $\eta_{\mathrm{p}}{ }^{2}=.21 ;\left(\right.$ all other $p$ 's $\left.>.16 ; \eta_{\mathrm{p}}{ }^{2} \mathrm{~s}<.11\right)$ 
Table A6 Slopes (ms/item) Experiment 6

\begin{tabular}{|c|c|c|c|c|}
\hline & \multicolumn{4}{|c|}{ Colour + Orientation } \\
\hline & \multicolumn{2}{|c|}{ Green Diagonal T } & \multicolumn{2}{|c|}{ Red Cardinal T } \\
\hline & Absent & Present & Absent & Present \\
\hline \multicolumn{5}{|l|}{ Eligibility } \\
\hline $100 \%$ & 54 & 13 & 59 & 19 \\
\hline $80 \%$ & 57 & 12 & 60 & 22 \\
\hline $60 \%$ & 43 & 11 & 51 & 13 \\
\hline $40 \%$ & 33 & 11 & 38 & 8 \\
\hline $20 \%$ & 15 & 4 & 13 & 8 \\
\hline
\end{tabular}

Experiment 7 - Motion + Orientation

Error Analysis

A four-way repeated measures ANOVA (eligibility $\mathrm{x}$ motion $\mathrm{x}$ display size $\mathrm{x}$ target) on the errors only found the expected result of more errors on target-present trials $F(1,15)=14.81$, $p=.002, \eta_{\mathrm{p}}{ }^{2}=.50$ and a smaller difference in errors between target-present and target-absent trials for smaller numbers of eligible items as can be seen from the significant two-way interaction between eligibility and target presence $F(3.4,50.96)=3.56, p=.017, \eta_{\mathrm{p}}{ }^{2}=.19$ (all other $p$ 's $\left.>.11 ; \eta_{\mathrm{p}}{ }^{2} \mathrm{~s}^{<}<.13\right)$.

Table A7 Slopes (ms/item) Experiment 7

\begin{tabular}{|c|c|c|c|c|}
\hline & \multicolumn{4}{|l|}{ Motion } \\
\hline & \multicolumn{2}{|c|}{ Static Diagonal T } & \multicolumn{2}{|c|}{ Moving Cardinal T } \\
\hline & Absent & Present & Absent & Present \\
\hline \multicolumn{5}{|l|}{ Eligibility } \\
\hline $100 \%$ & 67 & 19 & 71 & 14 \\
\hline $83 \%$ & 78 & 21 & 66 & 18 \\
\hline $67 \%$ & 61 & 16 & 69 & 26 \\
\hline $33 \%$ & 48 & 5 & 49 & 14 \\
\hline $17 \%$ & 37 & 9 & 31 & 14 \\
\hline
\end{tabular}


Differences in top-down guiding attributes 75

Acknowledgement

I would like to thank Victoria Swallow, Erin McWilliams and Kamelia Grozdeva for their assistance in data collection. 